\title{
Transport through vertical graphene contacts under intense laser fields
}

\author{
P. Stadler, T. Löfwander $\odot$, and M. Fogelström $\odot$ \\ Department of Microtechnology and Nanoscience (MC2), Chalmers University of Technology, S-412 96 Göteborg, Sweden
}

(Received 3 March 2020; revised manuscript received 11 May 2020; accepted 14 May 2020;

published 3 June 2020)

\begin{abstract}
We theoretically study the electronic and transport properties of two graphene layers vertically coupled by an insulating layer under the influence of a time-periodic external light field. The nonadiabatic driving induces excitations of electrons and a redistribution of the occupied states which are manifested in the opening of gaps in the quasienergy spectrum of graphene. When a voltage is applied between the top and bottom graphene layers, the photoinduced nonequilibrium occupation modifies the transport properties of the contact. We investigate the electronic and transport properties of the contact by using the nonequilibrium Green's function formalism. To illustrate the behavior of the differential conductance of the vertical contact under light illumination, we consider two cases. First, we assume that both the bottom and top layers consist of graphene and, second, we consider a finite mass term in the bottom layer. We obtain that the differential conductance is strongly suppressed due to opening of gaps in the quasienergy spectrum in graphene. Additionally, the conductance shows features corresponding to the tunneling of photoexcited electrons at energies of the Van Hove singularity for both the top and bottom layers. In the case of a finite mass term in the bottom layer, the differential conductance can be directly related to the tunneling of photoexcited electrons.
\end{abstract}

DOI: 10.1103/PhysRevResearch.2.023274

\section{INTRODUCTION}

The outstanding mechanical, optical, and electronic properties of graphene make it an attractive material for nextgeneration technology [1]. Prominent examples are applications of graphene in optoelectronic devices such as photodetectors [2-4] and sensors [5-7]. The core of the improved functionality in graphene-based optoelectronic devices lies in the interaction of light and matter in low dimensions. Although a remarkable absorption of $2.3 \%$ of incident light in monolayer graphene [8] is too weak to realize highperformance devices, several methods have been proposed to tune and enhance light-matter interaction in graphene. Among them, the absorption of graphene is enhanced by exciting surface plasmonic resonances [9-12] and by utilizing resonant structures to couple light with graphene [13-15].

Apart from employing light-matter interaction in graphene to enhance the functionality of optoelectronic devices, graphene reveals several fundamental light-induced phenomena [16]. One of these phenomena is the possibility to open gaps in the energy spectra by irradiating graphene with a timeperiodic potential [17-24]. When the frequency is smaller than the bandwidth of graphene, a so-called dynamical gap [17] opens at energies $\varepsilon=\hbar \omega / 2$ corresponding to a resonant

Published by the American Physical Society under the terms of the Creative Commons Attribution 4.0 International license. Further distribution of this work must maintain attribution to the author(s) and the published article's title, journal citation, and DOI. Funded by Bibsam. absorption/emission of a photon with frequency $\omega$ between the valence and conduction band.

The periodic driving is in general studied within Floquet theory $[25,26]$ and it was shown that topological trivial materials can be tuned into topological ones by a timeperiodic perturbation $[19,27,28]$. The topological properties of Floquet states in graphene have been extensively studied in Refs. [29-46] both without [29-40] and in presence of dissipation [41-46]. Floquet bands have been experimentally observed by time- and angle-resolved photoemission spectroscopy of surface Dirac fermions in a topological insulator under circular light irradiation [47,48] while photoinduced chiral edge states in graphene have been experimentally studied in Refs. [49,50]. Signature of light-matter interaction can also be detected by conductance measurements. The opening of band gaps due to light-matter interaction is manifested in a suppression of the conductance [22-24,51]. In contrast to a time-periodic external light field, time-dependent modulation of gate or contact potentials and the accompanied photoassisted tunneling of electrons lead to a great variety of interesting phenomena [52-57].

Motivated by the growing interest in nonequilibrium driving of graphene devices, we study a contact consisting of two graphene layers vertically separated by an insulating layer. A voltage is applied independently between the top and bottom layers allowing for tunneling between the layers. A similar setup consisting of a graphene/boron nitride/graphene heterostructure was discussed in Ref. [58] and it was demonstrated that resonant tunneling with the conservation of energy and momentum can be achieved by aligning the crystallographic orientation of the top and bottom graphene layers. In addition to the applied voltage, we assume that the top layer is driven to a nonequilibrium state by a time-periodic 
monochromatic light. We discuss two different scenarios. First, we assume that the vertical contact consists of two graphene layers. Second, we assume that a finite gap opens in the bottom layer. In our approach, we focus on noninteracting electrons in which Coulomb interaction is neglected. We study effects of both linear and circular polarization on the density of states, the occupation, and the differential conductance.

The main results are the following. The differential conductance is strongly suppressed in a broad range of voltages. In a qualitative picture, the suppression of the differential conductance is related to opening of gaps in the quasienergy spectrum due to time-dependent driving. Various aspects of the suppression of the conductance due to light-matter interaction have been discussed in Refs. [22-24,39,40,51]. Here, we extend these previous results of the differential conductance for vertical graphene contacts and the regime beyond the linear Dirac approximation.

For strong driving and high frequencies, the momentum dependence of the optical matrix elements and the nonlinearity of the graphene band structure becomes apparent in the differential conductance. Additional features occur at voltages that correspond to tunneling of photoexcited electrons to energies of the Van Hove singularity in the top or bottom layer. For the case of a finite mass term in the bottom layer, the signatures in the differential conductance can be directly related to the tunneling of photoexcited electrons. Although we restrict the discussion to light absorption in graphene, the approach can be extended to other two-dimensional materials [59].

The paper is structured as follows. In Sec. II, we introduce the continuous Hamiltonian and discuss the individual contribution of the Hamiltonian. From the continuous Hamiltonian, we derive a tight-binding Hamiltonian describing the lightmatter interaction and the tunneling between the contacts. In Sec. III we describe the nonequilibrium Green's function formalism and the relations of the Green's functions to the physical properties. Sections IV-VI contain the results of our paper. In Secs. IV and V we study the density of states, the nonequilibrium distribution function, and the occupation of graphene under light illumination, respectively. Finally, in Sec. VI, we discuss the differential conductance and show that the light-matter interaction induces suppression and enhancement of the conductance. In Sec. VII, we summarize and conclude.

\section{MODEL}

We consider a vertical contact consisting of a top $(t)$ and a bottom layer $(b)$ which are coupled by an insulating layer described by a tunneling element $t$ as shown in Fig. 1. A voltage $V$ is applied between the top and the bottom layers and an external electric field perpendicular to the layers induces a nonequilibrium occupation of the electronic states. For simplicity, we assume that the electric field interacts only with the top layer and that the sample size is much smaller than the wavelength of the light such that we can regard the light field as being uniform in space.

The Hamiltonian of the vertical junction is given by

$$
\hat{H}(t)=\hat{H}_{t}(t)+\hat{H}_{b}+\hat{H}_{\mathrm{tun}},
$$

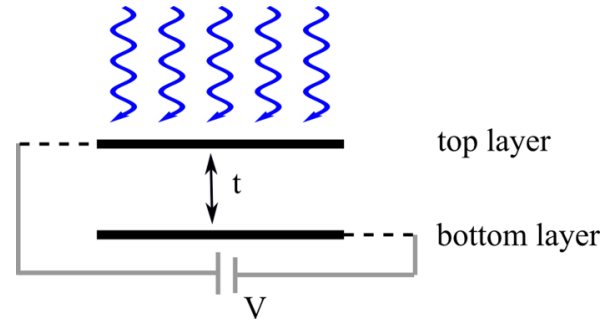

FIG. 1. Sketch of a contact with a top and bottom layer of graphene that are coupled by a tunneling element $t$. An electric field interacts with the top layer incident perpendicular to the layers. A voltage is applied between the top and the bottom layers.

with the Hamiltonians $\hat{H}_{t}(t)$ of the top layers, $\hat{H}_{b}$ of the bottom layer, and the tunneling Hamiltonian $\hat{H}_{\text {tun }}$. In the following, we discuss the contributions to $\hat{H}(t)$ separately. We introduce a continuous time-dependent Hamiltonian $\hat{H}_{t}(t)$ of the top layer under light illumination and discretize it to obtain a tight-binding Hamiltonian. Similar to the top layer, we then introduce the Hamiltonian of the bottom layer and the tunneling Hamiltonian.

\section{A. Hamiltonian of graphene under light illumination}

The Hamiltonian describing the motion of electrons with mass $m$ in the periodic lattice potential of graphene under light radiation can be written as

$$
\begin{aligned}
\hat{H}_{t}(t)= & \int d \boldsymbol{x} \hat{\Psi}_{t}^{\dagger}(\boldsymbol{x}) \\
& \times\left\{\frac{[\hat{\boldsymbol{p}}-e \boldsymbol{A}(t)]^{2}}{2 m}+\sum_{\boldsymbol{x}^{\prime}=\boldsymbol{x}_{A}, \boldsymbol{x}_{B}} V\left(\boldsymbol{x}-\boldsymbol{x}^{\prime}\right)\right\} \hat{\Psi}_{t}(\boldsymbol{x}),
\end{aligned}
$$

with the field operators of the upper layer $\hat{\Psi}_{t}^{\dagger}(\boldsymbol{x})$ and $\hat{\Psi}_{t}(\boldsymbol{x})$. The periodic lattice potential of graphene is composed of two triangular sublattice potentials $A$ and $B$, respectively. The light-matter coupling is obtained from the minimal substitution $\hat{\boldsymbol{p}} \rightarrow \hat{\boldsymbol{p}}-\boldsymbol{e A}(t)$ and the vector potential $\boldsymbol{A}(t)$ is modeled classically in the dipole approximation. In the following studies we consider the case of linear polarized light $\boldsymbol{A}(t)=\left(A_{x} \cos (\omega t), 0,0\right)$ and circular polarized light $\boldsymbol{A}(t)=$ $\left(A_{x} \cos (\omega t), A_{y} \sin (\omega t), 0\right)$ with the frequency $\omega$ of the photons.

We then transform the continuous Hamiltonian $\hat{H}_{t}(t)$ into a tight-binding Hamiltonian and expand the field operators in terms of the carbon $2 p_{z}$ wave functions $\Phi_{t}(\boldsymbol{x})$,

$$
\begin{aligned}
\hat{\Psi}_{t}(\boldsymbol{x})= & \frac{1}{\sqrt{N}} \sum_{\boldsymbol{k}_{t}, \boldsymbol{x}_{A}} e^{i \boldsymbol{k}_{t} \boldsymbol{x}_{A}} \Phi_{t}\left(\boldsymbol{x}-\boldsymbol{x}_{A}\right) \hat{a}_{\boldsymbol{k}_{t}} \\
& +\frac{1}{\sqrt{N}} \sum_{\boldsymbol{k}_{t}, \boldsymbol{x}_{B}} e^{i \boldsymbol{k}_{t} \boldsymbol{x}_{B}} \Phi_{t}\left(\boldsymbol{x}-\boldsymbol{x}_{B}\right) \hat{b}_{\boldsymbol{k}_{t}},
\end{aligned}
$$

with the annihilation operators $\hat{a}_{\boldsymbol{k}_{t}}$ and $\hat{b}_{\boldsymbol{k}_{t}}$ of a particle with momentum $\boldsymbol{k}_{t}$ on the sublattices $A$ and $B$ in the top layer, respectively.

The tight-binding Hamiltonian is separated into a bare Hamiltonian $\hat{H}_{0}$ and a Hamiltonian $\hat{H}_{I}(t)$ describing the interaction of graphene with the optical field $\hat{H}_{t}(t)=\hat{H}_{0}+\hat{H}_{I}(t)$. 

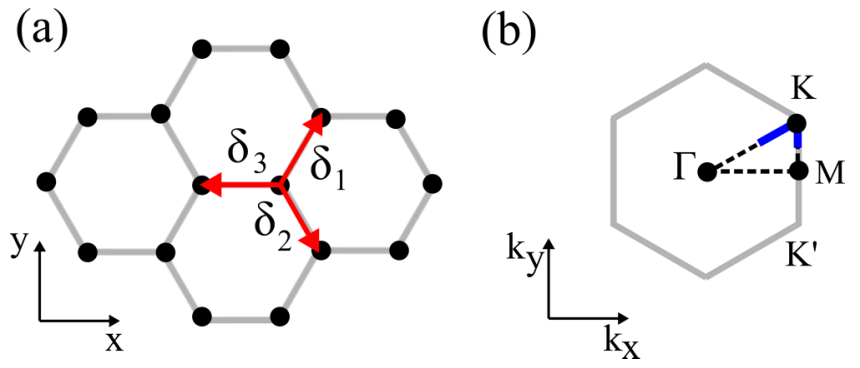

FIG. 2. (a) Lattice of graphene with the two sublattices $A$ (blue) and $B$ (green). The vectors $\boldsymbol{\delta}_{i}(i=1,2,3)$ connect the neighboring carbon atoms. (a) First Brillouin zone of graphene with the symmety points $\boldsymbol{\Gamma}, \boldsymbol{M}, \boldsymbol{K}$, and $\boldsymbol{K}^{\prime}$. The blue path indicates the momentum dependence in the following figures.

It is convenient to transform the operators in sublattice space to the space of the conduction and valence electrons. We write the creation operator of a quasiparticle in the conduction $(c)$ and valence $(v)$ band as $\hat{\psi}_{\boldsymbol{k}_{t}}^{\dagger}=\left(\hat{a}_{\boldsymbol{k}_{t}}^{c \dagger} \hat{a}_{\boldsymbol{k}_{t}}^{v \dagger}\right)$. The bare Hamiltonian can then be written as

$$
\hat{H}_{0}=\sum_{\boldsymbol{k}_{t}} \hat{\psi}_{\boldsymbol{k}_{t}}^{\dagger} \hat{\varepsilon}_{\boldsymbol{k}_{t}} \hat{\psi}_{\boldsymbol{k}_{t}}
$$

with the eigenenergies

$$
\hat{\varepsilon}_{\boldsymbol{k}_{t}}=\left(\begin{array}{cc}
\varepsilon_{\boldsymbol{k}_{t}} & 0 \\
0 & -\varepsilon_{\boldsymbol{k}_{t}}
\end{array}\right),
$$

and $\varepsilon_{\boldsymbol{k}_{t}}=\varepsilon_{\boldsymbol{k}_{t}}^{0}-\left(\mu_{t}-\varepsilon_{D}\right)$ with $\varepsilon_{\boldsymbol{k}_{t}}^{0}=-\gamma_{0}\left|f\left(\boldsymbol{k}_{t}\right)\right|$. The Dirac point energy $\varepsilon_{D}$ sets the energy of the Dirac point relative to the chemical potential $\mu$. The interaction energy between the next-nearest-neighbor carbon atoms is given by $\gamma_{0}$ and the chemical potential is $\mu_{t}$. In the above equation

$$
f\left(\boldsymbol{k}_{t}\right)=\sum_{i} e^{i \boldsymbol{k}_{t} \boldsymbol{\delta}_{i}}
$$

with the vectors $\delta_{i}$ connecting neighboring carbon atoms. These vectors are given by $\boldsymbol{\delta}_{1}=\frac{a}{2}(1, \sqrt{3}), \boldsymbol{\delta}_{2}=\frac{a}{2}(1,-\sqrt{3})$ and $\delta_{3}=(-1,0)$ with the nearest-neighbor distance between the atoms $a=1.42 \AA$. The vectors $\delta_{i}$ together with the lattice of graphene are shown in Fig. 2(a).

Following Refs. [60,61], we work in the Coulomb gauge $\boldsymbol{\nabla} \cdot \boldsymbol{A}=0$ and apply the dipole approximation since the momentum of the photon is negligible compared to the momentum of the electrons. The light-matter interaction can then be written as

$$
\hat{H}_{I}(t)=\sum_{\boldsymbol{k}_{t}} \hat{\psi}_{\boldsymbol{k}_{t}}^{\dagger} \hat{M}_{\boldsymbol{k}_{t}}(t) \hat{\psi}_{\boldsymbol{k}_{t}}
$$

with the light-matter interaction matrix

$$
\hat{M}_{\boldsymbol{k}_{t}}(t)=\left(\begin{array}{cc}
M_{\boldsymbol{k}_{t}}^{c c}(t) & M_{\boldsymbol{k}_{t}}^{c v}(t) \\
M_{\boldsymbol{k}_{t}}^{v c}(t) & M_{\boldsymbol{k}_{t}}^{v v}(t)
\end{array}\right),
$$

and the elements

$$
\begin{aligned}
& M_{\boldsymbol{k}}^{c c}(t)=\operatorname{Re}\left[\boldsymbol{M}_{\boldsymbol{k}}\right] \boldsymbol{A}(t)=-M_{\boldsymbol{k}}^{v v}(t), \\
& M_{\boldsymbol{k}}^{c v}(t)=i \operatorname{Im}\left[\boldsymbol{M}_{\boldsymbol{k}}\right] \boldsymbol{A}(t)=M_{\boldsymbol{k}}^{v c^{*}}(t) .
\end{aligned}
$$

The time-independent elements $\boldsymbol{M}_{\boldsymbol{k}}$ are

$$
\boldsymbol{M}_{\boldsymbol{k}}=i \frac{e}{m} \hbar M e^{-i \phi_{k}} \sum_{i} e^{i \boldsymbol{k} \boldsymbol{\delta}_{i}} \frac{\boldsymbol{\delta}_{i}}{\left|\boldsymbol{\delta}_{i}\right|}
$$

with $\phi_{\boldsymbol{k}}=\arg [f(\boldsymbol{k})]$ and a constant factor $M=$ $\int d \boldsymbol{x} \Phi_{u}(\boldsymbol{x}) \partial_{x} \Phi_{u}\left(\boldsymbol{x}-\left|\boldsymbol{\delta}_{i}\right| \hat{\boldsymbol{e}}_{x}\right)$ with the unit vector in the $x$ direction $\hat{\boldsymbol{e}}_{x}$.

The light-matter interaction can be divided into intraband coupling, $M_{\boldsymbol{k}_{l}}^{c c}(t)$ and $M_{\boldsymbol{k}_{l}}^{v v}(t)$, and interband coupling, $M_{\boldsymbol{k}_{l}}^{c v}(t)$ and $M_{\boldsymbol{k}_{l}}^{v c}(t)$. The intraband coupling describes processes in which an electron in the valence (conduction) band absorbs/emits a photon with frequency $\omega$ and stays in the valence (conduction) band. In a process of interband coupling, an electron scatters from the valence (conduction) band to the conduction (valence) band and exchanges energy of the photon.

In the following, we combine all the constant values of Eq. (11) in the amplitude of the vector potential $\boldsymbol{A}(t)$ and introduce the coupling strength

$$
\alpha_{x, y}=(e / m) \hbar M A_{x, y}
$$

in units of energy. To get an estimate of the magnitude of the vector potential, we consider the case of linear polarized light and convert the vector potential $\boldsymbol{A}(t)$ to an electric field by $\boldsymbol{E}(t)=\partial \boldsymbol{A}(t) / \partial t$. In Ref. [62], a graphene sample was illuminated with an electric field amplitude $E_{x}=4.0 \times 10^{7} \mathrm{~V} / \mathrm{m}$ [62] and a photon energy $\hbar \omega \approx 191 \mathrm{meV}$. Introducing the interaction energy of graphene $\gamma_{0}=2.6 \mathrm{eV}$ of the next-nearestneighbor carbon atoms, the photon energy corresponds to $\hbar \omega \approx 0.07 \gamma_{0}$. The coupling strength in Eq. (12) then is of the order $\alpha_{x, y} \approx 0.02 \gamma_{0}$ with the factor $M \simeq 3.0 \mathrm{~nm}^{-1}$ [61].

For the purpose of presentation, we show in the following sections the results for slightly larger electric fields and photon energies. For example, in Secs. IV-VI, we assume an electric field amplitude of the order of $E_{x}=0.1 \mathrm{~V} / \AA$ and a photon energy $\hbar \omega \approx 1.7 \mathrm{eV}$ corresponding to $\alpha_{x, y} \approx 0.1 \gamma_{0}$ and $\hbar \omega \approx$ $0.6 \gamma_{0}$. We remark that high frequencies pulses with energies $\hbar \omega \approx 1.7 \mathrm{eV}$ have been for instance applied in dielectrics to control the electric current by the electric field [63]. Although in our approach, we assume a constant external field, high electric field amplitude can only be achieved by laser pulses. However, as pointed out in Ref. [62], a constant field amplitude is still a valid approach if the envelope of the Gaussian pulse varies slowly in time $[62,64,65]$.

\section{B. Hamiltonian of the bottom lead and tunneling Hamiltonian}

The electrons can tunnel to the bottom layer which we model as graphene with a finite mass term. The Hamiltonian can be written as

$$
\hat{H}_{b}=\sum_{\boldsymbol{k}_{b}} \hat{\psi}_{\boldsymbol{k}_{b}}^{\dagger} \hat{\varepsilon}_{\boldsymbol{k}_{b}} \hat{\psi}_{\boldsymbol{k}_{b}}
$$

with the excitation energies $\varepsilon_{\boldsymbol{k}_{b}}=\varepsilon_{\boldsymbol{k}_{b}}^{0}-\left(\mu_{b}-\varepsilon_{D}\right), \varepsilon_{\boldsymbol{k}_{b}}^{0}=$ $-\gamma_{0} \sqrt{\left|f\left(\boldsymbol{k}_{b}\right)\right|^{2}+m_{b}^{2}}$, the chemical potential $\mu_{b}$, and the
mass $m_{b}$.

The tunneling Hamiltonian is given by

$$
\hat{H}_{\mathrm{tun}}=\sum_{\boldsymbol{k}_{b} \boldsymbol{k}_{t}} t_{\boldsymbol{k}_{t} \boldsymbol{k}_{b}} \hat{\psi}_{\boldsymbol{k}_{b}}^{\dagger} \hat{\psi}_{\boldsymbol{k}_{t}}+\text { H.c., }
$$


with the tunneling elements $\boldsymbol{t}_{\boldsymbol{k}_{t} \boldsymbol{k}_{b}}$. In the following, we assume momentum conservation at tunneling and set $t_{\boldsymbol{k} \boldsymbol{k}^{\prime}}=\delta_{\boldsymbol{k} \boldsymbol{k}^{\prime}} t$.

\section{METHOD}

Since we are interested in nonequilibrium transport properties, we apply the Keldysh Green's function approach to the Hamiltonian $\hat{H}$. In this method we express the physical properties in terms of Green's functions and calculate the corresponding Green's function [66,67]. To this end, we define the contour-ordered Green's function

$$
G_{\boldsymbol{k}}^{\lambda \lambda^{\prime}}\left(\tau, \tau^{\prime}\right)=-i\left\langle\mathcal{T} \hat{a}_{\boldsymbol{k}}^{\lambda}(\tau) \hat{a}_{\boldsymbol{k}}^{\lambda^{\prime \dagger}}\left(\tau^{\prime}\right)\right\rangle,
$$

with the indices $\lambda=(c, v)$ and $\lambda^{\prime}=(c, v)$ indicating either the conduction band (c) or the valence band $(v)$. The times $\tau$ and $\tau^{\prime}$ are located on the Keldysh contour and the contourordering operator is denoted as $\mathcal{T}$. To simplify the notation, we collect the Green's functions of the valence and conduction electrons in a matrix $\check{G}_{k}\left(\tau, \tau^{\prime}\right)$ defined by

$$
\hat{G}_{\boldsymbol{k}}\left(\tau, \tau^{\prime}\right)=\left(\begin{array}{ll}
G_{\boldsymbol{k}}^{c c}\left(\tau, \tau^{\prime}\right) & G_{\boldsymbol{k}}^{c v}\left(\tau, \tau^{\prime}\right) \\
G_{\boldsymbol{k}}^{v c}\left(\tau, \tau^{\prime}\right) & G_{\boldsymbol{k}}^{v v}\left(\tau, \tau^{\prime}\right)
\end{array}\right) .
$$

The general procedure then is to derive the Dyson equation for the Green's function in Eq. (16). This Green's function is given by

$$
\hat{G}_{k}\left(\tau, \tau^{\prime}\right)=\hat{g}_{k}\left(\tau, \tau^{\prime}\right)+\left(\hat{g}_{\boldsymbol{k}} \circ \hat{M}_{\boldsymbol{k}} \circ \hat{G}_{\boldsymbol{k}}\right)\left(\tau, \tau^{\prime}\right)
$$

with the convolution given by $(A \circ B)\left(\tau, \tau^{\prime}\right)=$ $\int d \tau_{1} A\left(\tau, \tau_{1}\right) B\left(\tau_{1}, \tau^{\prime}\right)$, the matrix $\hat{M}_{k}\left(\tau, \tau^{\prime}\right)=\hat{M}_{k}(\tau) \delta(\tau-$ $\left.\tau^{\prime}\right)$, and the Green's function

$$
\hat{g}_{k}\left(\tau, \tau^{\prime}\right)=\left(\begin{array}{cc}
g_{k}^{c}(\tau) & 0 \\
0 & g_{k}^{v}(\tau)
\end{array}\right) \delta\left(\tau-\tau^{\prime}\right),
$$

corresponding to the Hamiltonian $\hat{H}_{0}$. In the next step, the Dyson equation has to be transformed from the contour time to the real time $[66,67]$. After the transformation to the real time, every element in Eq. (16) becomes a matrix in Keldysh space defined by

$$
\check{G}_{\boldsymbol{k}}\left(t, t^{\prime}\right)=\left(\begin{array}{ll}
\hat{G}_{\boldsymbol{k}}^{11}\left(t, t^{\prime}\right) & \hat{G}_{\boldsymbol{k}}^{12}\left(t, t^{\prime}\right) \\
\hat{G}_{\boldsymbol{k}}^{21}\left(t, t^{\prime}\right) & \hat{G}_{\boldsymbol{k}}^{22}\left(t, t^{\prime}\right)
\end{array}\right) .
$$

In the above expression, the upper indices 1 or 2 refer to the position of the times $t$ and $t^{\prime}$ on the Keldysh contour. A definition of all the Green's functions in Eq. (19) and their relations is given in Appendix A. From the elements in Eq. (19) we can derive the retarded Green's function by $\hat{G}_{k}^{R}\left(t, t^{\prime}\right)=\hat{G}_{k}^{11}\left(t, t^{\prime}\right)-\hat{G}_{k}^{12}\left(t, t^{\prime}\right)$. The advanced Green's function is related to the retarded Green's function by its Hermitian conjugate $\hat{G}_{k}^{A}\left(t, t^{\prime}\right)=\hat{G}_{k}^{R^{\dagger}}\left(t^{\prime}, t\right)$. The retarded $(R)$, advanced $(A)$, and the lesser (12) Green's functions constitute the building blocks by which we will express the charge current in the following section.

\section{A. Green's functions without light irradiation}

Following the procedure that we describe, we derive the Green's function for the bottom lead. Since we assume that the light only interacts with the upper layer, these Green's functions correspond to the Hamiltonian in Eq. (13) with momentum $\boldsymbol{k}_{b}$. In Fourier space, the retarded Green's function is given by $g_{\boldsymbol{k}_{b}}^{c, R}(\varepsilon)=1 /\left(\varepsilon+i \eta-\varepsilon_{\boldsymbol{k}_{b}}\right)$ and $g_{\boldsymbol{k}_{b}}^{v, R}(\varepsilon)=1 /(\varepsilon+$ $i \eta+\varepsilon_{\boldsymbol{k}_{b}}$ ), with an infinitesimal small real part $\eta$. The lesser Green's function is $\hat{g}_{\boldsymbol{k}_{b}}^{12}(\varepsilon)=-\left[\hat{g}_{\boldsymbol{k}_{b}}^{R}(\varepsilon)-\hat{g}_{\boldsymbol{k}_{b}}^{A}(\varepsilon)\right] f_{b}(\varepsilon)$ with the temperature $T$ and the Fermi function $f_{b}(\varepsilon)=\{1+\exp [(\varepsilon-$ $\left.\left.\left.\mu_{b}\right) / k_{B} T\right]\right\}^{-1}$ with the chemical potential on the bottom layer $\mu_{b}$.

\section{B. Green's functions under light irradiation}

In this section, we derive the Dyson equation for the retarded and lesser Green's functions under light irradiation. Because of the time dependence in the optical field, we apply a discrete Fourier transformation to the Green's functions. To keep the notation simple, we replace the momentum $\boldsymbol{k}_{t}$ on the top layer with $\boldsymbol{k}$.

From Eq. (17) and the relations between the Green's functions in Keldsyh space we can derive the Dyson equation for the retarded Green's function

$$
\hat{G}_{\boldsymbol{k}}^{R}\left(t, t^{\prime}\right)=\hat{g}_{\boldsymbol{k}}^{R}\left(t, t^{\prime}\right)+\left(\hat{g}_{\boldsymbol{k}}^{R} \circ \hat{M}_{\boldsymbol{k}} \circ \hat{G}_{\boldsymbol{k}}^{R}\right)\left(t, t^{\prime}\right) .
$$

The Green's functions in Eq. (20) depend only on momentum $\boldsymbol{k}$ due to the dipole approximation in the light-matter interaction and the accompanied momentum conservation. Similarly, we can derive the lesser Green's function

$$
\hat{G}_{\boldsymbol{k}}^{12}\left(t, t^{\prime}\right)=\left(\hat{G}_{\boldsymbol{k}}^{R} \circ \hat{g}_{\boldsymbol{k}}^{R^{-1}} \circ \hat{g}_{\boldsymbol{k}}^{12} \circ \hat{g}_{\boldsymbol{k}}^{A^{-1}} \circ \hat{G}_{\boldsymbol{k}}^{A}\right)\left(t, t^{\prime}\right) .
$$

To compute the Green's functions in Eqs. (20) and (21) we have to apply a discrete Fourier transformation due to the time dependence of the light-matter interaction. We can solve the Dyson equations by first transforming the time arguments to the Wigner representation with the relative time $\tau=t-t^{\prime}$ and the center-of-mass time $T=\left(t+t^{\prime}\right) / 2$ [67]. Second, we apply the Fourier transformation [66]

$$
\hat{G}_{\boldsymbol{k}}^{R, 12}\left(t, t^{\prime}\right)=\sum_{n} \int \frac{d \varepsilon}{2 \pi} \hat{G}_{\boldsymbol{k}, n}^{R, 12}(\varepsilon) e^{-i \varepsilon \tau} e^{-i n \omega T}
$$

and then numerically solve the Dyson equation by matrix inversion. To simplify later equations, we introduce the notation for the Fourier-transformed Green's function $\hat{G}_{\boldsymbol{k}, n m}^{R, 12}(\varepsilon)=$ $\hat{G}_{\boldsymbol{k}, n-m}^{R, 12}[\varepsilon+(n+m) \omega / 2]$. After the Fourier transformation of Eq. (20) we obtain

$$
\begin{aligned}
\hat{G}_{\boldsymbol{k}, n m}^{R}(\varepsilon)= & \hat{g}_{\boldsymbol{k}, n}^{R}(\varepsilon) \delta_{n m}-\hat{g}_{\boldsymbol{k}, n}^{R}(\varepsilon) \hat{M}_{\boldsymbol{k}}^{+} \hat{G}_{\boldsymbol{k}, n+1 m}^{R}(\varepsilon) \\
& -\hat{g}_{\boldsymbol{k}, n}^{R}(\varepsilon) \hat{M}_{\boldsymbol{k}}^{-} \hat{G}_{\boldsymbol{k}, n-1 m}^{R}(\varepsilon)
\end{aligned}
$$

with $\hat{g}_{\boldsymbol{k}, n}^{R}(\varepsilon)=\hat{g}_{\boldsymbol{k}}^{R}(\varepsilon+n \omega)$ and the time-independent matrices

$$
\hat{M}_{\boldsymbol{k}}^{ \pm}=\left(\begin{array}{cc}
\operatorname{Re}\left[\boldsymbol{M}_{\boldsymbol{k}}\right] \boldsymbol{A}^{ \pm} & i \operatorname{Im}\left[\boldsymbol{M}_{\boldsymbol{k}}\right] \boldsymbol{A}^{ \pm} \\
-i \operatorname{Im}\left[\boldsymbol{M}_{\boldsymbol{k}}\right] \boldsymbol{A}^{ \pm} & -\operatorname{Re}\left[\boldsymbol{M}_{\boldsymbol{k}}\right] \boldsymbol{A}^{ \pm}
\end{array}\right) .
$$

In Eq. (24), $\boldsymbol{A}^{ \pm}$refers to the components of the vector potential $\boldsymbol{A}(t)=\boldsymbol{A}^{+} e^{i \omega t}+\boldsymbol{A}^{-} e^{-i \omega t}$ which are proportional to positive $(+)$ and negative $(-)$ frequencies, respectively. For the case of linear polarization the components are $\boldsymbol{A}^{ \pm}=$ $\left(A_{x} / 2,0,0\right)$ and for circular polarization $\boldsymbol{A}^{+}=\left(A_{x} / 2,0,0\right)$ and $\boldsymbol{A}^{-}=\left(0,-i A_{y} / 2,0\right)$. The unperturbed retarded Green's functions in Eq. (23) of the conduction and valence electrons are given by $g_{\boldsymbol{k}, n}^{c, R}(\varepsilon)=\left(\varepsilon+n \omega+i \eta-\varepsilon_{\boldsymbol{k}}\right)^{-1}$ and $g_{\boldsymbol{k}, n}^{v, R}(\varepsilon)=$ $\left(\varepsilon+n \omega+i \eta+\varepsilon_{k}\right)^{-1}$. 
Applying the Fourier transform of the lesser Green's function in Eq. (21) gives

$$
\hat{G}_{\boldsymbol{k}, n m}^{12}(\varepsilon)=\sum_{p} \hat{G}_{\boldsymbol{k}, n p}^{R}(\varepsilon) \hat{g}_{\boldsymbol{k}, p}^{R^{-1}}(\varepsilon) \hat{g}_{\boldsymbol{k}, p}^{12}(\varepsilon) \hat{g}_{\boldsymbol{k}, p}^{A^{-1}}(\varepsilon) \hat{G}_{\boldsymbol{k}, p m}^{A}(\varepsilon) .
$$

The unperturbed lesser Green's function is given by $\hat{g}_{\boldsymbol{k}, n}^{12}(\varepsilon)=-\left[\hat{g}_{\boldsymbol{k}, n}^{R}(\varepsilon)-\hat{g}_{\boldsymbol{k}, n}^{A}(\varepsilon)\right] f_{t, n}(\varepsilon)$ with the temperature $T$ and the Fermi function $f_{t, n}(\varepsilon)=\{1+\exp [(\varepsilon+n \omega-$ $\left.\left.\left.\mu_{t}\right) / k_{B} T\right]\right\}^{-1}$ with the chemical potential $\mu_{t}$ on the top layer.

When applying a Fourier transformation to the unperturbed Green's functions we have introduced a small imaginary part in in the energy to ensure convergence of the time integrals [66,67]. The parameter $\eta$ should be chosen such that the final results are independent of $\eta$. In later sections, we have set $\eta=$ $10^{-3}-10^{-4} \gamma_{0}$ to be the smallest parameter in our modeling.

\section{Charge current}

With the Green's function derived in the previous sections, we can calculate the charge current. The current on the bottom lead can be expressed as

$$
I_{b}=-\frac{2 e}{\hbar} \sum_{\boldsymbol{k}_{b} \boldsymbol{k}_{t}} \operatorname{Tr}\left\{\tilde{G}_{\boldsymbol{k}_{t} \boldsymbol{k}_{b}}^{12}(t, t) t_{\boldsymbol{k}_{b} \boldsymbol{k}_{t}}-t_{\boldsymbol{k}_{t} \boldsymbol{k}_{b}} \tilde{G}_{\boldsymbol{k}_{b} \boldsymbol{k}_{t}}^{12}(t, t)\right\},
$$

with the trace over the Green's functions in the space of the conduction and valence electrons. In Eq. (26) $\tilde{G}_{\boldsymbol{k}_{\boldsymbol{k}} \boldsymbol{k}_{b}}^{12}(t, t)$ is the Green's functions corresponding to the full Hamiltonian $\hat{H}(t)$ taking the coupling between the leads into account to infinite order in the tunneling. However, to simplify the calculations, we restrict the discussion to the tunneling limit for which we can write the current as

$$
I_{b}=\frac{2 e}{\hbar} \sum_{n} e^{i n \omega T} I_{b}^{n}
$$

with

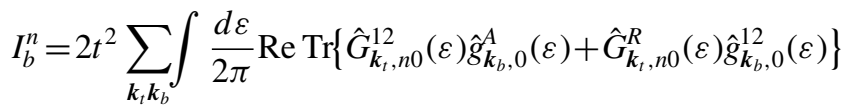

with $t_{k k^{\prime}}=\delta_{k k^{\prime}} t$ and the momentum-independent tunneling element $t$. Since the summations over $\boldsymbol{k}_{t}$ and $\boldsymbol{k}_{b}$ separate in the tunneling limit, we can compute the summation over $\boldsymbol{k}_{b}$ in the bottom layer analytically $[68,69]$. In Appendix $\mathrm{B}$, we present a recursive method to compute the Green's functions $\hat{G}_{\boldsymbol{k}, n m}^{R, A, 12}$.

\section{DENSITY OF STATES UNDER LIGHT IRRADIATION}

In this section, we discuss the density of states and quasienergy spectrum of the graphene under light irradiation. We derive an effective Hamiltonian to study the splitting of quasieigenstates of graphene due to the light-matter interaction. The light-matter interaction of graphene induces sidebands of the conduction and valence bands, which are shifted by $\pm n \omega$ from the eigenenergies $\varepsilon_{\boldsymbol{k}}$ of the bare graphene Hamiltonian. In the following, we restrict the discussion to a case of $n=4$ sidebands since the effect of light on the quasienergy spectrum decreases with the number of sidebands and higher orders will not modify the results.
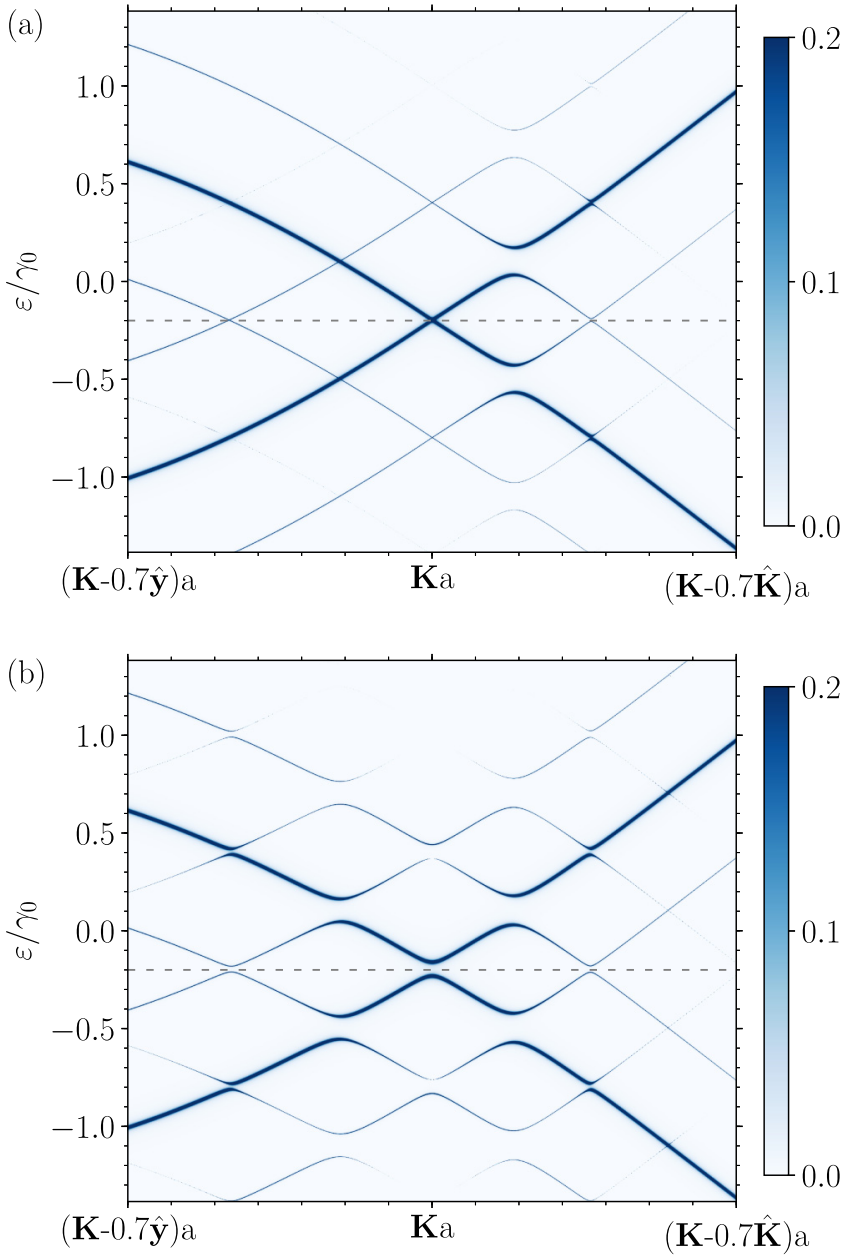

FIG. 3. Density of states $\rho_{\boldsymbol{k}}(\varepsilon)$ in Eq. (29) as a function of energy and momentum along the path $(\boldsymbol{K}-0.7 \hat{\boldsymbol{y}}) \rightarrow \boldsymbol{K} \rightarrow(\boldsymbol{K}-0.7 \hat{\boldsymbol{K}})$ in the first Brillouin zone. The path is shown in Fig. 2(b) as blue line. The unit vectors in $\boldsymbol{y}$ and $\boldsymbol{K}$ directions are written as $\hat{\boldsymbol{y}}$ and $\hat{\boldsymbol{K}}$, respectively. The dashed line shows that Dirac point energy which is set to $\varepsilon_{D}=-0.2 \gamma_{0}$. The frequency is $\omega=0.6 \gamma_{0}, n=4$, and $\eta=10^{-4} \gamma_{0}$. In (a) we consider linear polarized light with $\boldsymbol{A}(t)=\left(A_{x} \cos (\omega t), 0,0\right)$ and $\alpha_{x}=0.1 \gamma_{0}$. In this case the element $\Delta_{k}$ vanishes along the path $(\boldsymbol{K}-0.7 \hat{\boldsymbol{y}}) \rightarrow \boldsymbol{K}$ and the spectrum remains ungapped. In (b), we consider circular polarized light with $\boldsymbol{A}(t)=$ $\left(A_{x} \cos (\omega t), A_{y} \sin (\omega t), 0\right)$ and $\alpha_{x}=\alpha_{y}=0.1 \gamma_{0}$. The density of states shows gaps at finite energies and becomes fully gapped at the Dirac point energy. The splitting close to the energies $\varepsilon_{k}=\omega / 2+\varepsilon_{D}$ is given by $\Delta_{k}$.

The quasispectrum of graphene under light irradiation has, for example, been discussed in literature using Floquet theory $[19,29,30,70]$. For completeness and discussions in later sections, we present here the results of the density of states of the band structure for momenta in the first Brillouin zone using the approach discussed in Sec. III.

The momentum-dependent density of states $\rho_{k}(\varepsilon)$ of graphene under light irradiation is given by

$$
\rho_{\boldsymbol{k}}(\varepsilon)=-\frac{1}{2 \pi} \operatorname{Im} \operatorname{Tr} \hat{G}_{k, 00}^{R}(\varepsilon)
$$

with the retarded Green's function in Eq. (23). Figure 3 shows the density of states of graphene along the path $(\boldsymbol{K}-$ 
$0.7 \hat{\boldsymbol{y}}) \rightarrow \boldsymbol{K} \rightarrow(\boldsymbol{K}-0.7 \hat{\boldsymbol{K}})$ in the first Brillouin zone shown in Fig. 2(b). The unit vectors in $\boldsymbol{y}$ and $\boldsymbol{K}$ directions are written as $\hat{\boldsymbol{y}}$ and $\hat{\boldsymbol{K}}$, respectively. We discuss both the case of linear polarized light [Fig. 3(a)] and circularly polarized light [Fig. 3(b)]. Since a typical graphene sample is doped, we set the Dirac point energy to $\varepsilon_{D}=-0.2 \gamma_{0}$ such that states both above and below the Dirac point energy are occupied in an equilibrium state. The Dirac point energy in Fig. 3 is indicated by the dashed line.

The interaction of light with graphene induces opening of gaps, the most prominent one is called the dynamical gap [17] and occurs at energies $\varepsilon_{k}=\omega / 2+\varepsilon_{D}$. The transition corresponds to a resonant absorption/emission of a photon with energy $\hbar \omega$ between the conduction and valence bands. Importantly, the matrix elements $\hat{M}_{k}^{ \pm}$in Eq. (24) depend on momentum and on the polarization of the light. For certain momenta and polarization, the matrix element vanishes and a gap opening is suppressed. In the case of circularly polarized light, the quasienergies become fully gapped at the Dirac point energy. It has also been shown that the opening of a dynamical gap in graphene due to circularly polarized light is accompanied by a topological phase transition from a trivial to a topological band structure $[21,29,30]$.

To further study the effect of the light-matter interaction on graphene, we restrict the discussion to a minimal model, in which we consider the emission or absorption of one photon such that $n=0, \pm 1$. An effective Hamiltonian can be derived by comparing the retarded Green's function in Eq. (23) with the definition of a retarded Green's function $\hat{G}_{k, n m}^{R}(\varepsilon)=(\varepsilon+$ $\left.i \eta-\hat{H}_{k, n m}^{\text {eff }}\right)^{-1}$. We can then write the effective Hamiltonian as

$$
\hat{H}_{k, n m}^{\text {eff }}=\left(\begin{array}{ccc}
\hat{\varepsilon}_{\boldsymbol{k}}+\mathbb{1} \omega & -\hat{M}_{\boldsymbol{k}}^{+} & 0 \\
-\hat{M}_{\boldsymbol{k}}^{-} & \hat{\varepsilon}_{\boldsymbol{k}} & -\hat{M}_{\boldsymbol{k}}^{+} \\
0 & -\hat{M}_{\boldsymbol{k}}^{-} & \hat{\varepsilon}_{\boldsymbol{k}}-\mathbb{1} \omega
\end{array}\right) .
$$

Assuming that $\hat{M}_{k}^{ \pm}=0$, the eigenvalues of $\hat{H}_{k, n m}^{\text {eff }}$ are given by $E^{c, v}= \pm \varepsilon_{k}$ and the sidebands $E_{+}^{c, v}= \pm \varepsilon_{k}+\omega$ and $E_{-}^{c, v}=$ $\pm \varepsilon_{k}-\omega$ which are shifted by $\pm \omega$ from the original conduction and valence band, respectively.

To study the dynamical gap, we simplify the effective Hamiltonian and only consider the conduction band coupled with the first sideband of the valence band. Such an effective model corresponds to a $2 \times 2$ matrix in $\hat{H}_{k}^{\text {eff }}$ and describes the gap at finite energies. The eigenvalues for such a system are given by

$$
\varepsilon_{\boldsymbol{k}}^{ \pm}=\frac{\omega}{2} \pm \sqrt{\left(\varepsilon_{\boldsymbol{k}}-\frac{\omega}{2}\right)^{2}+\left|\Delta_{k}\right|^{2}}
$$

with the off-diagonal matrix element $\Delta_{k}=\left|\operatorname{Im}\left[\boldsymbol{M}_{k}\right] \boldsymbol{A}^{ \pm}\right|$being proportional to the absolute value of the imaginary part of the light-matter matrix element connecting the valence and conduction bands. Close to energies $\varepsilon_{k} \simeq \omega / 2+\varepsilon_{D}$, the splitting between the eigenvalues is therefore $2 \Delta_{k}$ and hence the gap is proportional to the coupling strength $\alpha_{x, y}$. The general behavior of gap openings in graphene under light illumination has been discussed in Ref. [70].

Since the dynamical gap is proportional to $\Delta_{k}$, we can understand the gap opening at finite energies in Fig. 3 by considering the momentum dependence of $\Delta_{k}$. Figure 4 shows the momentum dependence $\Delta_{\boldsymbol{k}}$ for (a) linear and (b) circular
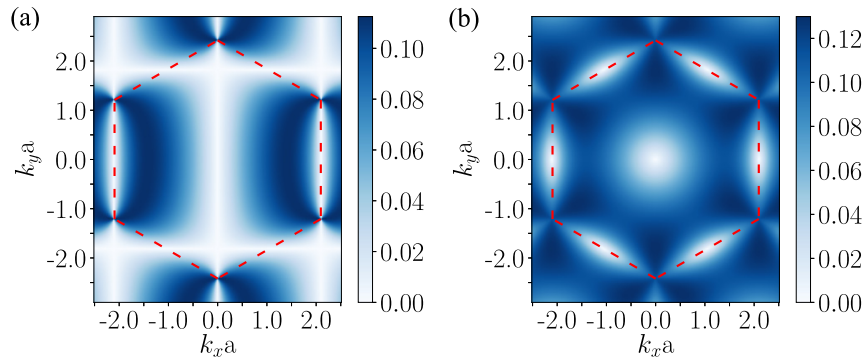

FIG. 4. Absolute value of the optical elements $\Delta_{k}=$ $\left|\operatorname{Im}\left[\boldsymbol{M}_{\boldsymbol{k}}\right] \boldsymbol{A}^{ \pm}\right|$for (a) linear polarized light and (b) circular polarized light. The parameters are the same as in Fig. 3. The red dashed line shows the boundary of the first Brillouin zone. In (a), the element vanishes along the path $\boldsymbol{M} \rightarrow \boldsymbol{K}$ and the density states remains ungapped as shown in Fig. 3(a). In (b) the the optical matrix element $\Delta_{k}$ is finite when approaching the $\boldsymbol{K}$ point and the density of states is fully gapped as shown in Fig. 3(b).

polarized light with the dashed line denoting the first Brillouin zone. When changing the momentum from $(\boldsymbol{K}-0.7 \hat{\boldsymbol{y}}) \rightarrow \boldsymbol{K}$ [see Fig. 3(a)], the element $\Delta_{k}$ stays zero and states will be ungapped along the path $(\boldsymbol{K}-0.7 \hat{\boldsymbol{y}}) \rightarrow \boldsymbol{K}$. However, the element $\Delta_{k}$ is finite when changing the momentum along the path $\boldsymbol{K} \rightarrow(\boldsymbol{K}-0.7 \hat{\boldsymbol{K}})$ and the spectrum is gapped. In Fig. 4(b), the element $\Delta_{\boldsymbol{k}}$ is finite when approaching the $\boldsymbol{K}$ point from both $(\boldsymbol{K}-0.7 \hat{\boldsymbol{y}})$ and $(\boldsymbol{K}-0.7 \hat{\boldsymbol{K}})$, and the spectrum is gapped at energies $\varepsilon_{\boldsymbol{k}} \simeq \omega / 2+\varepsilon_{D}$ close to the $\boldsymbol{K}$ point.

\section{ELECTRONIC OCCUPATION}

In this section, we discuss the modification of the occupation on the upper lead due to light irradiation. Direct measurement of the nonequilibrium occupation has been performed on the surface states of a topological insulator by time- and angle-resolved photoemission spectroscopy [47].

From the definition of the Green's functions, we can write the energy and $\boldsymbol{k}$-dependent occupation of the top layer as

$$
n_{k}(\varepsilon)=-i \operatorname{Tr} \hat{G}_{k, 00}^{12}(\varepsilon)
$$

with the lesser Green's function given by Eq. (25).

Figure 5 shows the occupation along the path $(\boldsymbol{K}-$ $0.7 \hat{\boldsymbol{y}}) \rightarrow \boldsymbol{K} \rightarrow(\boldsymbol{K}-0.7 \hat{\boldsymbol{K}})$ in the first Brillouin zone and as a function of energy $\varepsilon$ for the same parameters as in Fig. 4. We consider the case of linear [Fig. 5(a)] and circular polarized light [Fig. 5(b)] with a frequency of $\omega=0.6 \gamma_{0}$. We set the chemical potential to $\mu_{t}=0.15 \gamma_{0}$, the Dirac point energy to $\varepsilon_{D}=-0.2 \gamma_{0}$, and we consider $n=4$ sidebands. Without light-matter interaction and at zero temperature, states can be occupied to the chemical potential $\mu_{t}$. A finite coupling strength excites electrons and occupies states above the Fermi level $\mu_{t}$.

It is interesting to note that the gaps in Fig. 5(b) close to energies $\varepsilon \approx 0.1 \gamma_{0}$ are different in size. These gaps correspond to the resonant absorption or emission of a single photon with energy $\omega$. The different gap sizes occur due to the matrix elements shown in Fig. 4(b) whose magnitude is dependent on the direction from which the Dirac point is approached. The different sizes of the gap at finite energy vanish within the Dirac approximation of the band structure and the optical 

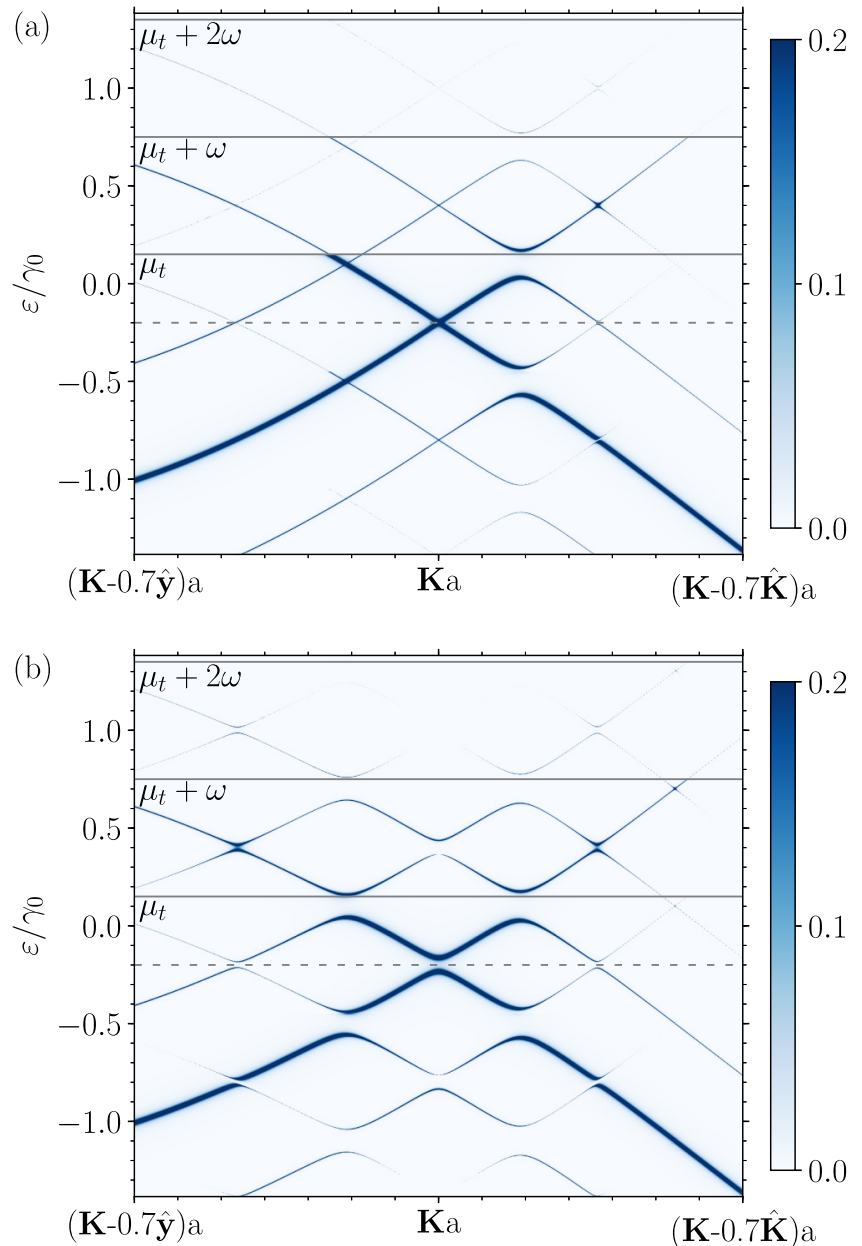

FIG. 5. Occupation of the top lead as a function of energy and momenta along the path $(\boldsymbol{K}-0.7 \hat{\boldsymbol{y}}) \rightarrow \boldsymbol{K} \rightarrow(\boldsymbol{K}-0.7 \hat{\boldsymbol{K}})$ in the first Brillouin zone. The path is shown in Fig. 2(b) as blue line. The frequency of the optical field is $\omega=0.6 \gamma_{0}$ and the chemical potentials are $\mu_{t}=0.15 \gamma_{0}, \varepsilon_{D}=-0.2 \gamma_{0}, n=4, T=0$, and $\eta=$ $10^{-4} \gamma_{0}$. Without the light-matter interaction, states are occupied at zero temperature to the chemical potential $\mu_{t}$. A finite optical field occupies states above the chemical potential with maximal energy $\mu_{t}+2 \omega$. In (a), the light is circular polarized with $\alpha_{x}=\alpha_{y}=0.1 \gamma_{0}$ and in (b) the light is linear polarized in the $x$ direction with $\alpha_{x}=$ $0.1 \gamma_{0}$ and $\alpha_{y}=0$.

matrix elements. The effect is a result of the consideration of the full momentum dependence of energies and optical matrix elements in the first Brillouin zone.

\section{Nonequilibrium distribution function}

The time-periodic external field drives graphene in a nonequilibrium state which is manifested by a nonequilibrium distribution function. The stationary nonequilibrium distribution is obtained from [67]

$$
F\left(\varepsilon_{\boldsymbol{k}}\right)=-\frac{i}{2 \pi} \begin{cases}\int_{-\infty}^{\infty} d \varepsilon \hat{G}_{\boldsymbol{k}, 00}^{12, v v}(\varepsilon), & \varepsilon_{\boldsymbol{k}}<\mu_{t} \\ \int_{-\infty}^{\infty} d \varepsilon \hat{G}_{\boldsymbol{k}, 00}^{12, c c}(\varepsilon), & \varepsilon_{\boldsymbol{k}}>\mu_{t} .\end{cases}
$$

Figure 6 shows the nonequilibrium distribution as a function of energy $\varepsilon_{\boldsymbol{k}}$ in state $\boldsymbol{k}$ along part of the path from the $\boldsymbol{\Gamma}$

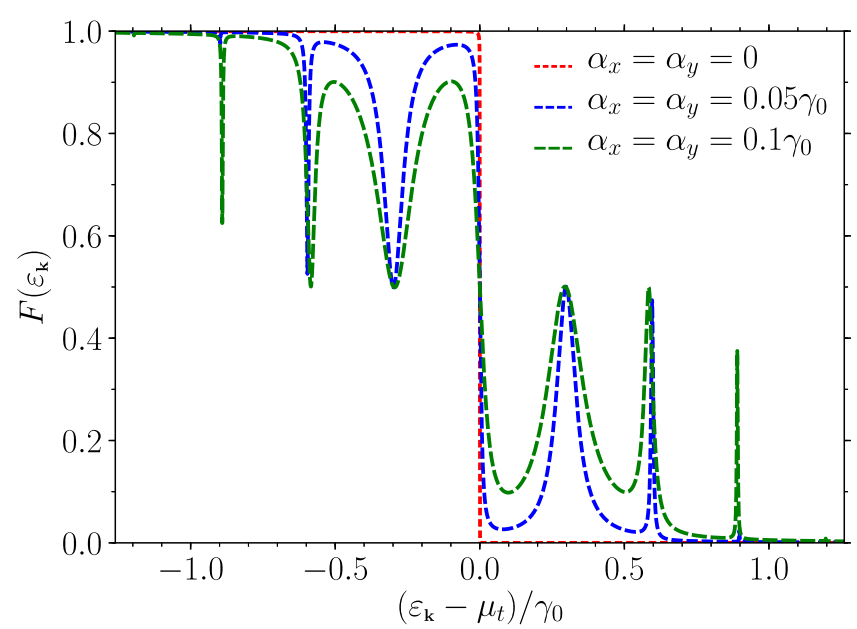

FIG. 6. Nonequilibrium distribution as a function of energy $\varepsilon_{\boldsymbol{k}}$ in state $\boldsymbol{k}$ along the path $(\boldsymbol{K}-0.7 \hat{\boldsymbol{K}}) \rightarrow \boldsymbol{K}$ in the first Brillouin zone. The frequencies of the optical field are $\omega=0.6 \gamma_{0}, n=4, T=0$, and $\eta=10^{-4} \gamma_{0}$. Without the light-matter interaction, the distribution function resembles the Fermi function at zero temperature. A finite circularly polarized field depletes quasienergy bands for $\varepsilon_{\boldsymbol{k}}<\mu_{t}$ and occupies states at $\varepsilon_{\boldsymbol{k}}>\mu_{t}$. The peaks and dips corresponds to resonance with $n=1,2,3$ and $n=4$ photons. The intensity decreases with the number of photons and the $n=4$ absorption process is strongly suppressed.

to the $\boldsymbol{K}$ point for different coupling strength and circularly polarized light. Without the light, the system is in equilibrium and the distribution function reduces to a Fermi function at zero temperature. A finite light-matter interaction redistributes the states depleting states in the valence band and occupying states in the conductance band [44]. The peaks and dips are at energies $\varepsilon_{\boldsymbol{k}}-\mu_{t}= \pm \omega / 2$ and correspond to a resonant absorption of a photon with $n=1,2,3$ and $n=4$ photons. The width of the dips and peaks depend on the coupling strength $\alpha_{x}$ and $\alpha_{y}$. Higher-order resonant processes become negligible small such that the resonant absorption of $n=4$ photons is barely visible even for the strong coupling $\alpha_{x}=$ $\alpha_{y}=0.1 \gamma_{0}$.

\section{DIFFERENTIAL CONDUCTANCE}

With the current derived in Eq. (27), we can discuss different scenarios for transport through a graphene contact and identify signatures of the light-matter interaction in transport measurements. We focus on the differential conductance which can be written as

$$
G(V)=\frac{d I_{b}^{0}(V)}{d V},
$$

with the dc current given by Eq. (27). Throughout the discussion, we assume that circularly polarized photons are absorbed in the top layer and the photoexcited electrons tunnel to the bottom layer.

Motivated by the fact that an underlying substrate [71] or Coulomb interaction [60] opens a gap in the band structure of graphene, we discuss two scenarios: (i) we assume that both the top and the bottom layers consist of graphene. (ii) We discuss the case that the top layer consists of graphene 

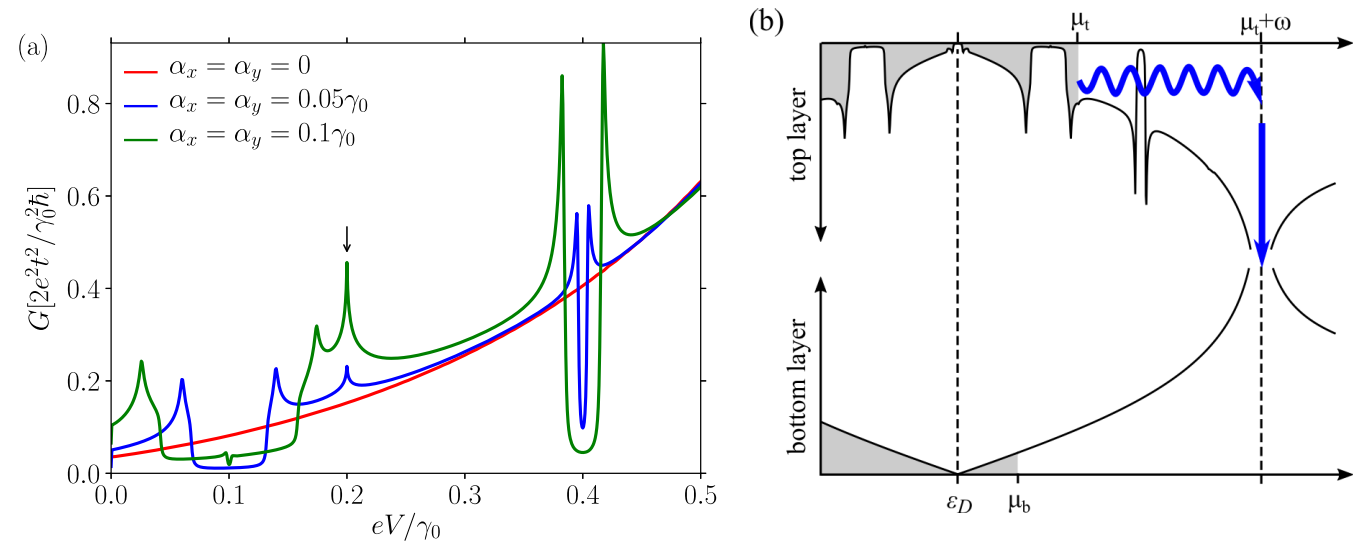

FIG. 7. (a) Differential conductance for circularly polarized light and different coupling strength $\alpha_{x}$ and $\alpha_{y}$. The parameters are $\omega=0.6 \gamma_{0}$, $T=0, n=4, \eta=10^{-3} \gamma_{0}$. The Dirac point energy is set to $\varepsilon_{D}=-0.2 \gamma_{0}$ such that the differential conductance is suppressed close to voltages $e V \simeq \omega / 2+\varepsilon_{D}=0.1 \gamma_{0}$. The light-matter interaction empties states and the differential conductance decreases. The steps in the case of $\alpha_{x}=\alpha_{y}=0.1 \gamma_{0}$ close to $e V \approx 0.03 \gamma_{0}$ and $e V \approx 0.17 \gamma_{0}$ are a signature of the nonlinear band structure of graphene for $\boldsymbol{k} \gg\left(\boldsymbol{K}, \boldsymbol{K}^{\prime}\right)$ and the momentum dependence of the optical matrix elements. (b) Sketch of the density of states of the top and bottom layers showing the process of photoexcitation of an electron at energy $\mu_{t}$. The photoexcited electron has energy matching the Van Hove singularity of the top layer and tunnels in the Van Hove singularity of the bottom layer. The process gives rise to a peak in (a) indicated by the black arrow.

and the bottom layer consists of graphene with a finite mass term opening a gap in the band structure.

\section{A. Tunneling between graphene contacts}

Figure 7(a) shows the differential conductance for circularly polarized light and different values of the coupling strength $\alpha_{x, y}$. The frequency of the incoming light is set to $\omega=$ $0.6 \gamma_{0}$ and the temperature is $T=0$. Since a typical graphene sample is doped we assume a finite Dirac point energy of $\varepsilon_{D}=-0.2 \gamma_{0}$ relative to the chemical potential $\mu_{t}$.

We first consider the case without light-matter interaction $\left(\alpha_{x}=\alpha_{y}=0\right)$. At small voltages $e V \ll \gamma_{0}$, the differential conductance increases with the applied voltage and depends nonlinearly on the voltage for $\mathrm{eV} \rightarrow \gamma_{0}$. Since we consider the tunneling current in Eq. (27) this behavior is reflected by the overlap between the density of states of the top and bottom layers. The density of states of single-layer graphene is proportional to $\varepsilon$ for $\varepsilon \ll \gamma_{0}$ and approaches the Van Hove singularity at $\varepsilon=0.8 \gamma_{0}$ assuming finite doping $\varepsilon_{D}=-0.2 \gamma_{0}$ [72].

As discussed in the previous section, a finite coupling strength opens a gap in the quasienergy spectrum of graphene. Even for moderate coupling strength $\alpha_{x}=\alpha_{y}=0.05 \gamma_{0}$, the opening of a gap in the quasienergy spectrum is manifested in a strong suppression of the differential conductance at certain voltages.

The first suppression of the current close to $\mathrm{eV}=0.1 \gamma_{0}$ corresponds to resonant absorption of a single photon between the conduction and valence bands. Since the Dirac point energy is at $\varepsilon=-0.2 \gamma_{0}$ and the frequency of the light is $\omega=0.6 \gamma_{0}$, the suppression of the conductance occurs at $e V \approx \omega / 2+\varepsilon_{D}=0.1 \gamma_{0}$. The second strong suppression of the differential conductance occurs close to $e V=0.4 \gamma_{0}$ and corresponds to resonant absorption of two photons between the conduction and valence bands.

At strong coupling $\alpha_{x}=\alpha_{y}=0.1 \gamma_{0}$, the differential conductance is suppressed over a larger range of voltages com- pared to the moderate coupling strength $\alpha_{x}=\alpha_{y}=0.05 \gamma_{0}$ since the gap in the quasienergy spectrum increases with increasing coupling strength. Interestingly, the suppression and the enhancement of the conductance close to voltages $e V \approx$ $0.1 \gamma_{0}$ gradually decreases and increases at strong coupling $\alpha_{x}=\alpha_{y}=0.1 \gamma_{0}$. The steps are a signature of the nonlinear band structure of graphene and the momentum dependence of the optical matrix elements for momenta $\boldsymbol{k} \gg\left(\boldsymbol{K}, \boldsymbol{K}^{\prime}\right)$. The gradual increase and decrease can be understood by considering, for instance, the conductance at voltage $\mathrm{eV}=0.15 \gamma_{0}$ in Fig. 7(a). Figure 5(b) shows the occupation corresponding to the voltage $e V=0.15 \gamma_{0}$ and the same parameters as in Fig. 7(a). Approaching the $\boldsymbol{K}$ point from $(\boldsymbol{K}-0.7 \hat{\boldsymbol{y}})$, states are occupied close to the chemical potential $\mu_{t}$. Starting from $e V=0.15 \gamma_{0}$ and increasing the voltage, first states close to $\boldsymbol{k}$ values $(\boldsymbol{K}-0.2 \hat{\boldsymbol{y}})$ contribute to the current and give rise to the first steplike increase of the conductance. Second states close to the $(\boldsymbol{K}-0.2 \hat{\boldsymbol{K}})$ are occupied by increasing the voltage and the conductance gradually increases a second time. A similar argument holds for the suppression of the conductance close to voltages $\mathrm{eV}=0.4 \gamma_{0}$ which is related to the resonant absorption of two photons.

In addition to a suppression of the differential conductance, the light-matter interaction induces a peak at $\mathrm{eV}=0.2 \gamma_{0}$ [black arrow in Fig. 7(a)]. The process corresponding to the peak is sketched in Fig. 7(b) and is related to absorption of a single photon at energy $\mu_{t}=0.2 \gamma_{0}$. Figure $7(\mathrm{~b})$ shows the density of states summed over all momenta in the first Brillouin zone of the top and bottom layers. In the top layer, the states are occupied to the chemical potential $\mu_{t}$. The lightmatter interaction excites an electron to energies $\varepsilon=\mu_{t}+\omega$ matching the energy of the Van Hove singularity of the top layer. The excited electron then tunnels to the Van Hove singularity of the bottom layer providing a large density of unoccupied states. The peak at $\mathrm{eV}=0.2 \gamma_{0}$ in the conductance is hence a result of the overlap of the Van Hove singularities of both the top and the bottom layer. 

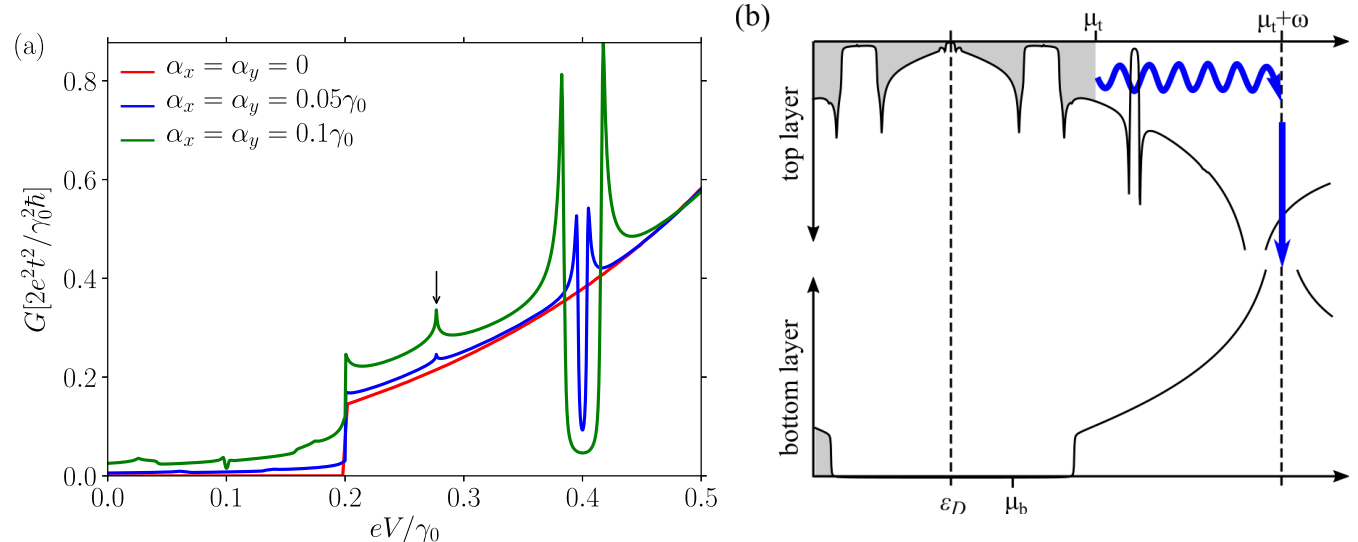

FIG. 8. (a) Differential conductance for circularly polarized light and different coupling strength $\alpha_{x}$ and $\alpha_{y}$. The parameters are $\omega=0.6 \gamma_{0}$, $\varepsilon_{D}=-0.2 \gamma_{0}, n=4, T=0, \eta=10^{-3} \gamma_{0}$. In comparison to Fig. 7(a), here we assume that the bottom layer consists of graphene with a finite mass term $m_{b}=0.4 \gamma_{0}$ which opens a gap of $2 m_{b}$. For $\alpha_{x}=\alpha_{y}=0$, the conductance jumps to a finite value when the voltage reaches the gap edge of the bottom graphene layer. Because of the Dirac point energy, the steplike increase sets in at $e V=0.2 \gamma_{0}$. A finite light-matter interaction excites electrons above the gap edge of the bottom lead and the conductance is finite even for voltages $\mathrm{eV}<0.2 \gamma_{0}$. (b) Sketch of the density of states of the top and bottom layers showing the process of absorption of a photon at energy $\mu_{t}$ and the accompanied tunneling in the Van Hove singularity of the bottom layer. The process gives rise to the peak at $e V=0.28 \gamma_{0}$ which is indicated in (a) by the black arrow. Because of the gap in the density of states of the bottom layer, the Van Hove singularity is shifted to larger energies compared to the energy of the Van Hove singularity of the top layer.

\section{B. Tunneling between graphene and graphene with finite mass term}

So far, we discussed the differential conductance for the ideal case that light is absorbed in the top layer. Since the light is only partially absorbed in graphene [8], also the lower layer will interact with the light. However, because of a substrate or interaction in graphene [60,71], a band gap will open. In this section, we assume that the band gap of the bottom layer is larger than the frequency of the light such that the lower layer becomes essentially transparent. In a more general setting, the bottom layer can be made of any two-dimensional material with a band gap larger than the frequency of the light. In Figs. 8(a) and 9, we consider a finite mass $m_{b}$ in the bottom layer such that the energy of the bare graphene Hamiltonian is given by $\varepsilon_{\boldsymbol{k}_{b}}=-\gamma_{0} \sqrt{\left|f\left(\boldsymbol{k}_{b}\right)\right|^{2}+m_{b}^{2}}-\left(\mu_{b}-\varepsilon_{D}\right)$ and the band gap becomes $2 m_{b}$.

Figure 8(a) shows the differential conductance with the same parameters as in Fig. 7(a) except that now the bottom layer has a mass term $m_{b}=0.4 \gamma_{0}$ and the band gap is $0.8 \gamma_{0}$. Without the light $\alpha_{x}=\alpha_{y}=0$, the current is completely suppressed at voltages $e V<m_{b}$ because of the band gap in the bottom layer. For $e V>0.2 \gamma_{0}$, the voltage is larger than the band gap on the bottom graphene and electrons can tunnel between the layers. We remark that the Dirac point energy is at $\varepsilon_{D}=-0.2 \gamma_{0}$ such that the gap edge on the bottom layer is reached for voltages $e V=0.2 \gamma_{0}$. When the voltage is larger than the gap of the bottom layer, the behavior is similar to the differential conductance in Fig. 7(a).

As we have seen in previous sections, a finite light-matter interaction can occupy states above the chemical potential. We expect that the photoexcited electrons contribute to a current although the voltage is smaller than the gap on the bottom lead. This behavior is seen in Fig. 8(a) at finite light-matter coupling strength. The differential conductance for $\mathrm{eV}<$
$0.2 \gamma_{0}$ is solely due to the tunneling of photoexcited electrons. However, since the energies of electrons that are excited by a single photon are smaller than the band-gap edge at $\mathrm{eV}=$ $0.2 \gamma_{0}$ of the bottom layer, the current is strongly suppressed for $e V<0.2 \gamma_{0}$. In this case, the two-photon absorption is the dominant process to the conductance.

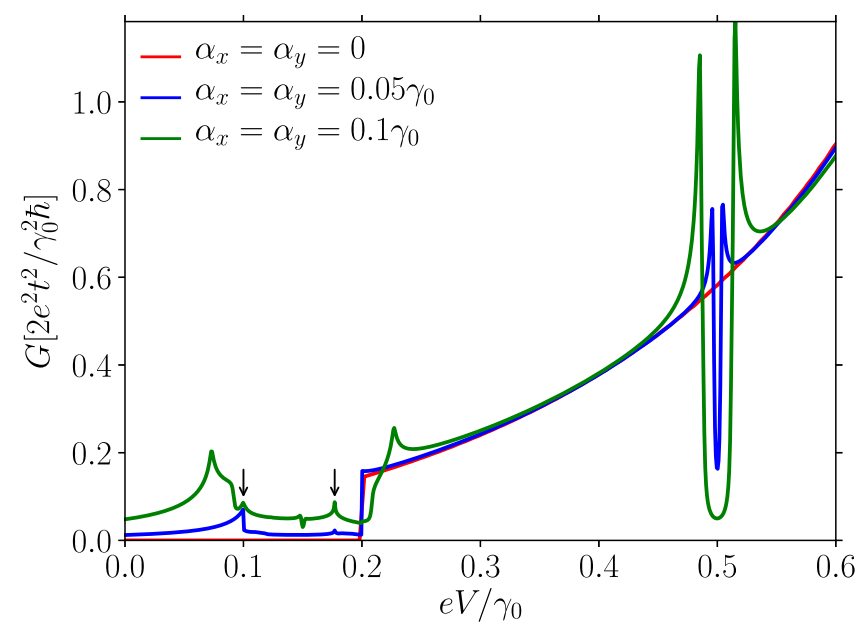

FIG. 9. Differential conductance for circular polarized light and different coupling strength $\alpha_{x}$ and $\alpha_{y}$. Compared to Fig. 8(a), the frequency is $\omega=0.7 \gamma_{0}$. The other parameters are $m_{b}=0.4 \gamma_{0}, n=4$, $T=0, \eta=10^{-3} \gamma_{0}$. The Dirac point energy is set to $\varepsilon_{D}=-0.2 \gamma_{0}$ such that the differential conductance is suppressed close to voltages $e V \simeq \omega / 2+\varepsilon_{D}=0.2 \gamma_{0}$. The finite conductance in the gap depends on the $\eta=0.001 \gamma_{0}$. The steps in the case of $\alpha_{x}=\alpha_{y}=0.1 \gamma_{0}$ are a signature of the nonlinear band structure of graphene for $\boldsymbol{k} \gg$ $\left(\boldsymbol{K}, \boldsymbol{K}^{\prime}\right)$. The two black arrows indicate the tunneling processes which are associated with the tunneling of photoexcited electrons to energies of the Van Hove singularity of either the top or bottom layer. 
Additionally to the finite conductance at voltages $\mathrm{eV}<$ $0.2 \gamma_{0}$, the differential conductance shows a peak at $e V \approx$ $0.28 \gamma_{0}$ indicated by the black arrow in Fig. 8(a). The process corresponding to such a peak is sketched in Fig. 8(b) and its origin is similar to the peak at $e V=0.2 \gamma_{0}$ in Fig. 7(a), namely, electrons at the chemical potential are photoexcited and can tunnel into a large number of empty states due to the Van Hove singularity of the bottom layer. The finite mass term in the bottom layer shifts the Van Hove singularity to higher energies such that the peak in Fig. 8(a) appears at $e V \approx 0.28 \gamma_{0}$. In comparison to Fig. 7(b), the energies of the photoexcited electrons at energy $\varepsilon \approx 0.88 \gamma_{0}$ are larger than the Van Hove singularity of the top layer but match the energy of the Van Hove singularity of the bottom layer.

In Fig. 9 we discuss the differential conductance at higher frequency $\omega=0.7 \gamma_{0}$ and the same parameters as in Fig. 8(a). In this case, the conductance shows complex features even below the band gap $\mathrm{eV}<0.2 \gamma_{0}$. Because of the high frequency $\omega=0.7 \gamma_{0}$ and the opening of the gap in the quasienergy spectrum of graphene, the absorption of a single photon is sufficient to excite an electron above the band-gap edge of the bottom layer. The resonant absorption of a single photon gives then rise to the increase of the conductance at voltages $\mathrm{eV} \approx 0.08 \gamma_{0}$.

Additionally, two smaller peaks occur at voltages $\mathrm{eV}=$ $0.1 \gamma_{0}$ and $0.18 \gamma_{0}$ indicated by the black arrows in Fig. 9. The origin of both peaks can be associated with the Van Hove singularities of both the top and the bottom layers. The Van Hove singularity of the bottom layer occurs at energy $\varepsilon=$ $0.8 \gamma_{0}$ due to the Dirac point energy $\varepsilon_{D}=-0.2 \gamma_{0}$. However, similar to Fig. 8(b), the Van Hove singularity of the bottom layer appears at energies $\varepsilon \approx 0.88 \gamma_{0}$ because of the finite mass term $m_{b}$. When an electron at voltage $e V=0.1 \gamma_{0}$ absorbs a photon, it is excited to energies of the Van Hove singularity of the top layer and then tunnels to the bottom layer. The opposite process is also possible, corresponding to first the excitation of an electron at voltages $e V=0.18 \gamma_{0}$, and second, the tunneling to the energy of the Van Hove singularity of the bottom layer.

\section{CONCLUSION}

We studied the transport properties of two graphene layers vertically coupled with an insulating layer. A voltage is applied between the top and the bottom layer giving rise to a tunneling current. In such a contact, we discussed the differential conductance when the top graphene layer is driven to a nonequilibrium state by an external light field.

In agreement with previous results, multiple gaps open in the quasienergy spectrum of graphene depending on the polarization. We obtained that the gaps corresponding to a resonant absorption/emission of a single photon between the conduction and valence bands have different sizes due to the momentum dependence of the optical matrix element. This effect becomes in particular important at frequencies $\omega \gtrsim$ $0.5 \gamma_{0}$ where the nonlinearity of the band structure of graphene can not be neglected.

We studied the differential conductance in two kinds of contacts. First, we considered the differential conductance between two graphene layers. Second, we studied the modi- fication of the conductance when a gap opens in the bottom layer. In both cases, the effects of the light-matter interaction are manifested in a strong suppression of the conductance at voltages $\mathrm{eV}=n \omega / 2+\varepsilon_{D}$ due to the opening of gaps in the quasienergy spectrum of graphene. In the case of strong driving, the different gap sizes at energies $\varepsilon=n \omega / 2+\varepsilon_{D}$ become apparent in the differential conductance. Additional peaks in the differential conductance can be related to the tunneling of photoexcited electrons to the energies of the Van Hove singularity of the top or bottom layer. For the case of a finite band gap in the bottom layer, the differential conductance is solely due to the photoexcited electrons and is hence a direct signature of the light-matter interaction. Finally, we remark that although we studied the tunneling between two graphene layers, our approach can be extended to tunneling between arbitrary two-dimensional materials.

We conclude with neglected interaction and an outlook about extension to our approach. The light-matter interaction in graphene has been subject of much research and the nonequilibrium driving of graphene has been investigated in various approaches. Most of these approaches focus on the noninteracting picture in which the Coulomb interaction is neglected. However, it has been shown that the electronelectron interaction in two-dimensional materials can be tuned by engineering the surrounding dielectric environment. The electron-electron interaction has a significant effect on the band structure. For instance, Coulomb interaction leads to an open of a gap in the band structure and the formation of excitons $[60,73]$.

The continuous applications of a laser pulse or light field to graphene results in heating and eventually damage of the sample. Under an intense laser pulse, the electron-phonon coupling in graphene will excite phonons and relax the electronic system. The phonon dissipation in the graphene under light irradiation and the effect on the transport properties can be studied by coupling the electronic system to a bath of phonons similar to the approaches in Refs. [41-46].

\section{ACKNOWLEDGMENTS}

We acknowledge S. Lara-Avila and H. He for interesting discussions and comments. This work was supported by the Swedish Foundation for Strategic Research (GMT14-0077).

\section{APPENDIX A: KELDYSH GREEN'S FUNCTIONS}

In this Appendix, we recall the definitions of the Green's function and summarize the main steps to calculate the retarded, advanced, and lesser Green's functions. We refer to the books of Refs. [66,67] for a detailed introduction.

Using the contour-ordered Green's function defined in Eq. (15), we derive the Dyson equation by using the Heisenberg equation of motion. We then transform the contourordered Green's functions to the real time and define the Green's function in Keldysh space as

$$
G_{k k}^{\lambda}\left(t, t^{\prime}\right)=\left(\begin{array}{ll}
G_{k k}^{\lambda, 11}\left(t, t^{\prime}\right) & G_{k k}^{\lambda, 12}\left(t, t^{\prime}\right) \\
G_{k k}^{\lambda, 21}\left(t, t^{\prime}\right) & G_{k k}^{\lambda, 22}\left(t, t^{\prime}\right)
\end{array}\right) .
$$

In the above expression, the upper indices 1 or 2 refer to the position of the times $t$ and $t^{\prime}$ on the Keldysh contour. In 
addition to the Green's function in Eq. (19), we define the retarded Green's function $G_{k \boldsymbol{k}}^{\lambda, R}\left(t, t^{\prime}\right)$ and the advanced Green's function $G_{\boldsymbol{k} \boldsymbol{k}}^{\lambda, A}\left(t, t^{\prime}\right)$.

These Green's functions and the ones in Eq. (19) are defined as

$$
\begin{gathered}
G_{\boldsymbol{k} \boldsymbol{k}^{\prime}}^{\lambda, 11}\left(t, t^{\prime}\right)=-i\left\langle\mathcal{T} \hat{a}_{\boldsymbol{k}}^{\lambda}(t) \hat{a}_{\boldsymbol{k}^{\prime}}^{\lambda^{\dagger}}\left(t^{\prime}\right)\right\rangle, \\
G_{\boldsymbol{k} \boldsymbol{k}^{\prime}}^{\lambda, 22}\left(t, t^{\prime}\right)=-i\left\langle\tilde{\mathcal{T}} \hat{a}_{\boldsymbol{k}}^{\lambda}(t) \hat{a}_{\boldsymbol{k}^{\prime}}^{\lambda^{\dagger}}\left(t^{\prime}\right)\right\rangle, \\
G_{\boldsymbol{k} \boldsymbol{k}^{\prime}}^{\lambda, 12}\left(t, t^{\prime}\right)=i\left\langle\hat{a}_{\boldsymbol{k}^{\prime}}^{\lambda^{\dagger}}\left(t^{\prime}\right) \hat{a}_{\boldsymbol{k}}^{\lambda}(t)\right\rangle, \\
G_{\boldsymbol{k} \boldsymbol{k}^{\prime}}^{\lambda, 21}\left(t, t^{\prime}\right)=-i\left\langle\hat{a}_{\boldsymbol{k}}^{\lambda}(t) \hat{a}_{\boldsymbol{k}^{\prime}}^{\lambda^{\dagger}}\left(t^{\prime}\right)\right\rangle, \\
G_{\boldsymbol{k} \boldsymbol{k}^{\prime}}^{\lambda, R}\left(t, t^{\prime}\right)=-i \theta\left(t-t^{\prime}\right)\left\langle\left\{\hat{a}_{\boldsymbol{k}}^{\lambda}(t), \hat{a}_{\boldsymbol{k}^{\prime}}^{\lambda^{\dagger}}\left(t^{\prime}\right)\right\}\right\rangle, \\
G_{\boldsymbol{k} \boldsymbol{k}^{\prime}}^{\lambda, A}\left(t, t^{\prime}\right)=i \theta\left(t^{\prime}-t\right)\left\langle\left\{\hat{a}_{\boldsymbol{k}}^{\lambda}(t), \hat{a}_{\boldsymbol{k}^{\prime}}^{\lambda^{\dagger}}\left(t^{\prime}\right)\right\}\right\rangle .
\end{gathered}
$$

The real-time-ordering and anti-time-ordering operators are denoted by $\mathcal{T}$ and $\tilde{\mathcal{T}}$, respectively. The anticommutator is denoted by $\{\ldots, \ldots\}$.

The Green's functions satisfy the following relations which are useful to derive the retarded and advanced Green's functions. The Green's functions are related by

$$
\begin{aligned}
G_{\boldsymbol{k} \boldsymbol{k}^{\prime}}^{\lambda, R}\left(t, t^{\prime}\right)-G_{\boldsymbol{k} \boldsymbol{k}^{\prime}}^{\lambda, A} & \left(t, t^{\prime}\right)=G_{\boldsymbol{k} \boldsymbol{k}^{\prime}}^{\lambda,>}\left(t, t^{\prime}\right)-G_{\boldsymbol{k} \boldsymbol{k}^{\prime}}^{\lambda,<}\left(t, t^{\prime}\right), \\
G_{\boldsymbol{k} \boldsymbol{k}^{\prime}}^{\lambda, K}\left(t, t^{\prime}\right) & =G_{\boldsymbol{k} \boldsymbol{k}^{\prime}}^{\lambda, 11}\left(t, t^{\prime}\right)+G_{\boldsymbol{k} \boldsymbol{k}^{\prime}}^{\lambda, 22}\left(t, t^{\prime}\right) \\
& =G_{\boldsymbol{k} \boldsymbol{k}^{\prime}}^{\lambda,<}\left(t, t^{\prime}\right)+G_{\boldsymbol{k} \boldsymbol{k}^{\prime}}^{\lambda,>>}\left(t, t^{\prime}\right), \\
G_{\boldsymbol{k} \boldsymbol{k}^{\prime}}^{\lambda, R}\left(t, t^{\prime}\right)= & G_{\boldsymbol{k} \boldsymbol{k}^{\prime}}^{\lambda, 11}\left(t, t^{\prime}\right)-G_{\boldsymbol{k} \boldsymbol{k}^{\prime}}^{\lambda,<}\left(t, t^{\prime}\right) \\
= & G_{\boldsymbol{k} \boldsymbol{k}^{\prime}}^{\lambda,>}\left(t, t^{\prime}\right)-G_{\boldsymbol{k} \boldsymbol{k}^{\prime}}^{\lambda, 22}\left(t, t^{\prime}\right) \\
G_{\boldsymbol{k} \boldsymbol{k}^{\prime}}^{\lambda, A}\left(t, t^{\prime}\right) & =G_{\boldsymbol{k} \boldsymbol{k}^{\prime}}^{\lambda,<}\left(t, t^{\prime}\right)-G_{\boldsymbol{k} \boldsymbol{k}^{\prime}}^{\lambda, 22}\left(t, t^{\prime}\right) \\
& =G_{\boldsymbol{k} \boldsymbol{k}^{\prime}}^{\lambda, 11}\left(t, t^{\prime}\right)-G_{\boldsymbol{k} \boldsymbol{k}^{\prime}}^{\lambda,>}\left(t, t^{\prime}\right) \\
G_{\boldsymbol{k} \boldsymbol{k}^{\prime}}^{\lambda, 11}\left(t, t^{\prime}\right) & =G_{\boldsymbol{k} \boldsymbol{k}^{\prime}}^{\lambda, R}\left(t, t^{\prime}\right)+G_{\boldsymbol{k} \boldsymbol{k}^{\prime}}^{\lambda,<}\left(t, t^{\prime}\right) \\
& =G_{\boldsymbol{k} \boldsymbol{k}^{\prime}}^{\lambda, A}\left(t, t^{\prime}\right)+G_{\boldsymbol{k} \boldsymbol{k}^{\prime}}^{\lambda,>}\left(t, t^{\prime}\right) \\
G_{\boldsymbol{k} \boldsymbol{k}^{\prime}}^{\lambda, 22}\left(t, t^{\prime}\right) & =G_{\boldsymbol{k} \boldsymbol{k}^{\prime}}^{\lambda,<}\left(t, t^{\prime}\right)-G_{\boldsymbol{k} \boldsymbol{k}^{\prime}}^{\lambda, A}\left(t, t^{\prime}\right) \\
& =G_{\boldsymbol{k} \boldsymbol{k}^{\prime}}^{\lambda,>}\left(t, t^{\prime}\right)-G_{\boldsymbol{k} \boldsymbol{k}^{\prime}}^{\lambda, R}\left(t, t^{\prime}\right)
\end{aligned}
$$

and

$$
\begin{aligned}
& G_{\boldsymbol{k} \boldsymbol{k}^{\prime}}^{\lambda,<}\left(t, t^{\prime}\right)=\left[G_{\boldsymbol{k} \boldsymbol{k}^{\prime}}^{\lambda, K}\left(t, t^{\prime}\right)-G_{\boldsymbol{k} \boldsymbol{k}^{\prime}}^{\lambda, R}\left(t, t^{\prime}\right)+G_{\boldsymbol{k} \boldsymbol{k}^{\prime}}^{\lambda, A}\left(t, t^{\prime}\right)\right] / 2, \\
& G_{\boldsymbol{k} \boldsymbol{k}^{\prime}}^{\lambda,>}\left(t, t^{\prime}\right)=\left[G_{\boldsymbol{k} \boldsymbol{k}^{\prime}}^{\lambda, K}\left(t, t^{\prime}\right)+G_{\boldsymbol{k} \boldsymbol{k}^{\prime}}^{\lambda, R}\left(t, t^{\prime}\right)-G_{\boldsymbol{k} \boldsymbol{k}^{\prime}}^{\lambda, A}\left(t, t^{\prime}\right)\right] / 2 .
\end{aligned}
$$

Further, the Hermitian conjugate of the electron Green's functions satisfies the relations

$$
\begin{aligned}
G_{\boldsymbol{k} \boldsymbol{k}^{\prime}}^{\lambda, R}\left(t, t^{\prime}\right)^{*} & =G_{\boldsymbol{k}^{\prime} \boldsymbol{k}}^{\lambda, A}\left(t^{\prime}, t\right), \\
G_{\boldsymbol{k} \boldsymbol{k}^{\prime}}^{\lambda,<}\left(t, t^{\prime}\right)^{*} & =-G_{\boldsymbol{k}^{\prime} \boldsymbol{k}}^{\lambda,<}\left(t^{\prime}, t\right), \\
G_{\boldsymbol{k} \boldsymbol{k}^{\prime}}^{\lambda,>}\left(t, t^{\prime}\right)^{*} & =-G_{\boldsymbol{k}^{\prime} \boldsymbol{k}}^{\lambda,>}\left(t^{\prime}, t\right), \\
G_{\boldsymbol{k} \boldsymbol{k}^{\prime}}^{\lambda, 11}\left(t, t^{\prime}\right)^{*} & =-G_{\boldsymbol{k}^{\prime} \boldsymbol{k}}^{\lambda, 22}\left(t^{\prime}, t\right), \\
G_{\boldsymbol{k} \boldsymbol{k}^{\prime}}^{\lambda, 22}\left(t, t^{\prime}\right)^{*} & =-G_{\boldsymbol{k}^{\prime} \boldsymbol{k}}^{\lambda, 11}\left(t^{\prime}, t\right) .
\end{aligned}
$$

\section{APPENDIX B: RECURSIVE SOLUTION OF GREEN'S FUNCTIONS}

As discussed in Sec. III, the retarded, advanced, and lesser Green's functions are the basic building blocks to calculate the dc current in Eq. (27). In this Appendix, we describe the recursive method to compute the retarded Green's functions $\hat{G}_{k, n m}^{R}(\varepsilon)$ in Eq. (23) following Refs. [74,75]. The advanced Green's function is related to the retarded Green's function by $\hat{G}_{\boldsymbol{k}, n m}^{A}(\varepsilon)=\hat{G}_{\boldsymbol{k}, m n}^{R^{\dagger}}(\varepsilon)$ and the lesser Green's function is obtained from Eq. (25).

From the relation of the Green's functions $\hat{G}_{\boldsymbol{k}, n m}^{R}(\varepsilon)=$ $\hat{G}_{\boldsymbol{k}, n-m}^{R, 12}[\varepsilon+(n+m) \omega / 2]$, one can show that $\hat{G}_{\boldsymbol{k}, n m}^{R}(\varepsilon)=$ $\hat{G}_{k, n-m 0}^{R}(\varepsilon+m \omega)$. In the following, it is hence sufficient to set $m=0$ and define the transfer matrices $\hat{z}_{n}^{ \pm}$by (neglecting the energy and momentum dependence)

$$
\hat{G}_{n 0}^{R}=\hat{z}_{n}^{ \pm} \hat{G}_{n \mp 10}^{R},
$$

where $\hat{z}_{n}^{+}$and $\hat{z}_{n}^{-}$are defined for $n>1$ and $n<-1$, respectively. Using the recursive equation of the retarded Green's function [Eq. (23)]

$$
\hat{G}_{n 0}^{R}=\hat{g}_{n}^{R} \delta_{n 0}-\hat{g}_{n}^{R} \hat{M}^{+} \hat{G}_{n+10}^{R}-\hat{g}_{n}^{R} \hat{M}^{-} \hat{G}_{n-10}^{R},
$$

it follows that the transfer matrices satisfy the relation

$$
\hat{z}_{n}^{ \pm}=-\left(\hat{g}_{n}^{R^{-1}}+M^{ \pm} \hat{z}_{n+1}^{ \pm}\right)^{-1} M^{\mp} .
$$

The recursive method then works as follows. Starting from large $n$, we set the elements $\hat{z}_{n+1}^{ \pm}$and $\hat{z}_{-n-1}^{ \pm}$to zero, and calculate the remaining transfer matrices $\hat{z}_{n}^{+}, \ldots, \hat{z}_{1}^{+}$and $\hat{z}_{-n}^{-}, \ldots, \hat{z}_{-1}^{-}$by Eq. (B3). Setting $n=0$ in Eq. (B2) and using Eq. (B1), the Green's function $\hat{G}_{00}^{R}$ is given by

$$
\hat{G}_{00}^{R}=\left(\hat{g}_{0}^{R^{-1}}+M^{+} \hat{z}_{1}^{+}+M^{-} \hat{z}_{-1}^{-}\right)^{-1} .
$$

To obtain the Green's functions for arbitrary $n$, we use the solution of $\hat{G}_{00}^{R}$ and apply Eq. (B1).
[1] A. C. Ferrari, F. Bonaccorso, V. Fal'ko, K. S. Novoselov, S. Roche, P. Bøggild, S. Borini, F. H. L. Koppens, V. Palermo, N. Pugno et al., Science and technology roadmap for graphene, related two-dimensional crystals, and hybrid systems, Nanoscale 7, 4598 (2015).
[2] T. Mueller, F. Xia, and P. Avouris, Graphene photodetectors for high-speed optical communications, Nat. Photonics 4, 297 (2010).

[3] F. Withers, T. H. Bointon, M. F. Craciun, and S. Russo, All graphene photodetectors, ACS Nano 7, 5052 (2013). 
[4] C.-H. Liu, Y.-C. Chang, T. B. Norris, and Z. Zhong, Graphene photodetectors with ultra-broadband and high responsivity at room temperature, Nat. Nanotechnol. 9, 273 (2014).

[5] F. Schedin, E. Lidorikis, A. Lombardo, V. G. Kravets, A. K. Geim, A. N. Grigorenko, K. S. Novoselov, and A. C. Ferrari, Surface-enhanced raman spectroscopy of graphene, ACS Nano 4, 5617 (2010).

[6] S.-K. Lee, H. Y. Jang, S. Jang, E. Choi, B. H. Hong, J. Lee, S. Park, and J.-H. Ahn, All graphene-based thin film transistors on flexible plastic substrates, Nano Lett. 12, 3472 (2012).

[7] R. Pearce, J. Eriksson, T. Iakimov, L. Hultman, A. Lloyd Spetz, and R. Yakimova, On the differing sensitivity to chemical gating of single and double layer epitaxial graphene explored using scanning kelvin probe microscopy, ACS Nano 7, 4647 (2013).

[8] R. R. Nair, P. Blake, A. N. Grigorenko, K. S. Novoselov, T. J. Booth, T. Stauber, N. M. R. Peres, and A. K. Geim, Fine structure constant defines visual transparency of graphene, Science 320, 1308 (2008).

[9] L. Ju, B. Geng, J. Horng, C. Girit, M. Martin, Z. Hao, H. A. Bechtel, X. Liang, A. Zettl, Y. R. Shen et al., Graphene plasmonics for tunable terahertz metamaterials, Nat. Nanotechnol. 6, 630 (2011).

[10] S. Thongrattanasiri, F. H. L. Koppens, and F. J. García de Abajo, Complete Optical Absorption in Periodically Patterned Graphene, Phys. Rev. Lett. 108, 047401 (2012).

[11] A. N. Grigorenko, M. Polini, and K. S. Novoselov, Graphene plasmonics, Nat. Photonics 6, 749 (2012).

[12] S. Kim, M. S. Jang, V. W. Brar, K. W. Mauser, L. Kim, and H. A. Atwater, Electronically tunable perfect absorption in graphene, Nano Lett. 18, 971 (2018).

[13] A. Ferreira, N. M. R. Peres, R. M. Ribeiro, and T. Stauber, Graphene-based photodetector with two cavities, Phys. Rev. B 85, 115438 (2012).

[14] M. Hashemi, M. H. Farzad, N. Asger Mortensen, and S. Xiao, Enhanced absorption of graphene in the visible region by use of plasmonic nanostructures, J. Opt. 15, 055003 (2013).

[15] B. Zhao, J. M. Zhao, and Z. M. Zhang, Enhancement of nearinfrared absorption in graphene with metal gratings, Appl. Phys. Lett. 105, 031905 (2014).

[16] M. Glazov and S. Ganichev, High frequency electric field induced nonlinear effects in graphene, Phys. Rep. 535, 101 (2014).

[17] S. V. Syzranov, M. V. Fistul, and K. B. Efetov, Effect of radiation on transport in graphene, Phys. Rev. B 78, 045407 (2008).

[18] F. J. López-Rodríguez and G. G. Naumis, Analytic solution for electrons and holes in graphene under electromagnetic waves: Gap appearance and nonlinear effects, Phys. Rev. B 78, 201406(R) (2008).

[19] T. Oka and H. Aoki, Photovoltaic Hall effect in graphene, Phys. Rev. B 79, 081406(R) (2009).

[20] O. V. Kibis, Metal-insulator transition in graphene induced by circularly polarized photons, Phys. Rev. B 81, 165433 (2010).

[21] S. E. Savel'ev and A. S. Alexandrov, Massless Dirac fermions in a laser field as a counterpart of graphene superlattices, Phys. Rev. B 84, 035428 (2011).

[22] H. L. Calvo, H. M. Pastawski, S. Roche, and L. E. F. F. Torres, Tuning laser-induced band gaps in graphene, Appl. Phys. Lett. 98, 232103 (2011).
[23] H. L. Calvo, P. M. Perez-Piskunow, S. Roche, and L. E. F. Foa Torres, Laser-induced effects on the electronic features of graphene nanoribbons, Appl. Phys. Lett. 101, 253506 (2012).

[24] H. L. Calvo, P. M. Perez-Piskunow, H. M. Pastawski, S. Roche, and L. E. F. Foa Torres, Non-perturbative effects of laser illumination on the electrical properties of graphene nanoribbons, J. Phys.: Condens. Matter 25, 144202 (2013).

[25] J. H. Shirley, Solution of the Schrödinger equation with a hamiltonian periodic in time, Phys. Rev. 138, B979 (1965).

[26] H. Sambe, Steady states and quasienergies of a quantummechanical system in an oscillating field, Phys. Rev. A 7, 2203 (1973).

[27] N. H. Lindner, G. Refael, and V. Galitski, Floquet topological insulator in semiconductor quantum wells, Nat. Phys. 7, 490 (2011).

[28] T. Kitagawa, E. Berg, M. Rudner, and E. Demler, Topological characterization of periodically driven quantum systems, Phys. Rev. B 82, 235114 (2010).

[29] P. M. Perez-Piskunow, G. Usaj, C. A. Balseiro, and L. E. F. Foa Torres, Floquet chiral edge states in graphene, Phys. Rev. B 89, 121401(R) (2014).

[30] G. Usaj, P. M. Perez-Piskunow, L. E. F. Foa Torres, and C. A. Balseiro, Irradiated graphene as a tunable Floquet topological insulator, Phys. Rev. B 90, 115423 (2014).

[31] P. M. Perez-Piskunow, L. E. F. Foa Torres, and G. Usaj, Hierarchy of Floquet gaps and edge states for driven honeycomb lattices, Phys. Rev. A 91, 043625 (2015).

[32] Y. Tenenbaum Katan and D. Podolsky, Generation and manipulation of localized modes in Floquet topological insulators, Phys. Rev. B 88, 224106 (2013).

[33] T. Kitagawa, T. Oka, A. Brataas, L. Fu, and E. Demler, Transport properties of nonequilibrium systems under the application of light: Photoinduced quantum Hall insulators without landau levels, Phys. Rev. B 84, 235108 (2011).

[34] M. Sentef, M. Claassen, A. Kemper, B. Moritz, T. Oka, J. Freericks, and T. Devereaux, Theory of Floquet band formation and local pseudospin textures in pump-probe photoemission of graphene, Nat. Commun. 6, 7047 (2015).

[35] A. Kundu, H. A. Fertig, and B. Seradjeh, Effective Theory of Floquet Topological Transitions, Phys. Rev. Lett. 113, 236803 (2014).

[36] M. Yang, Z.-J. Cai, R.-Q. Wang, and Y.-K. Bai, Topologically trivial and nontrivial edge bands in graphene induced by irradiation, Phys. Lett. A 380, 2836 (2016).

[37] Z. Gu, H. A. Fertig, D. P. Arovas, and A. Auerbach, Floquet Spectrum and Transport through an Irradiated Graphene Ribbon, Phys. Rev. Lett. 107, 216601 (2011).

[38] M. Fruchart, P. Delplace, J. Weston, X. Waintal, and D. Carpentier, Probing (topological) Floquet states through DC transport, Physica E: Low-dimensional Systems and Nanostructures 75, 287 (2016).

[39] SK Firoz Islam and A. Saha, Driven conductance of an irradiated semi-dirac material, Phys. Rev. B 98, 235424(R) (2018)

[40] L. E. F. Foa Torres, P. M. Perez-Piskunow, C. A. Balseiro, and G. Usaj, Multiterminal Conductance of a Floquet Topological Insulator, Phys. Rev. Lett. 113, 266801 (2014).

[41] H. Dehghani, T. Oka, and A. Mitra, Dissipative Floquet topological systems, Phys. Rev. B 90, 195429 (2014). 
[42] H. Dehghani, T. Oka, and A. Mitra, Out-of-equilibrium electrons and the Hall conductance of a Floquet topological insulator, Phys. Rev. B 91, 155422 (2015).

[43] H. Dehghani and A. Mitra, Optical Hall conductivity of a Floquet topological insulator, Phys. Rev. B 92, 165111 (2015).

[44] H. Dehghani and A. Mitra, Occupation probabilities and current densities of bulk and edge states of a Floquet topological insulator, Phys. Rev. B 93, 205437 (2016).

[45] T. Iadecola, T. Neupert, and C. Chamon, Occupation of topological Floquet bands in open systems, Phys. Rev. B 91, 235133 (2015).

[46] Q. Chen, L. Du, and G. A. Fiete, Floquet band structure of a semi-Dirac system, Phys. Rev. B 97, 035422 (2018).

[47] Y. H. Wang, H. Steinberg, P. Jarillo-Herrero, and N. Gedik, Observation of Floquet-bloch states on the surface of a topological insulator, Science 342, 453 (2013).

[48] F. Mahmood, C.-K. Chan, Z. Alpichshev, D. Gardner, Y. Lee, P. A. Lee, and N. Gedik, Selective scattering between Floquetbloch and Volkov states in a topological insulator, Nat. Phys. 12, 306 (2016).

[49] J. Karch, P. Olbrich, M. Schmalzbauer, C. Zoth, C. Brinsteiner, M. Fehrenbacher, U. Wurstbauer, M. M. Glazov, S. A. Tarasenko, E. L. Ivchenko et al., Dynamic Hall Effect Driven by Circularly Polarized Light in a Graphene Layer, Phys. Rev. Lett. 105, 227402 (2010).

[50] J. Karch, C. Drexler, P. Olbrich, M. Fehrenbacher, M. Hirmer, M. M. Glazov, S. A. Tarasenko, E. L. Ivchenko, B. Birkner, J. Eroms et al., Terahertz Radiation Driven Chiral Edge Currents in Graphene, Phys. Rev. Lett. 107, 276601 (2011).

[51] J. Atteia, J. H. Bardarson, and J. Cayssol, Ballistic transport through irradiated graphene, Phys. Rev. B 96, 245404 (2017).

[52] Y. Korniyenko, O. Shevtsov, and T. Löfwander, Resonant second-harmonic generation in a ballistic graphene transistor with an ac-driven gate, Phys. Rev. B 93, 035435 (2016).

[53] Y. Korniyenko, O. Shevtsov, and T. Löfwander, Nonlinear response of a ballistic graphene transistor with an ac-driven gate: High harmonic generation and terahertz detection, Phys. Rev. B 94, 125445 (2016).

[54] J. Hammer and W. Belzig, Scattering approach to frequencydependent current noise in Fabry-Pérot graphene devices, Phys. Rev. B 87, 125422 (2013).

[55] M. H. Pedersen and M. Büttiker, Scattering theory of photonassisted electron transport, Phys. Rev. B 58, 12993 (1998).

[56] G. Platero and R. Aguado, Photon-assisted transport in semiconductor nanostructures, Phys. Rep. 395, 1 (2004).

[57] S. Kohler, J. Lehmann, and P. Hänggi, Driven quantum transport on the nanoscale, Phys. Rep. 406, 379 (2005).

[58] A. Mishchenko, J. S. Tu, Y. Cao, R. V. Gorbachev, J. R. Wallbank, M. T. Greenaway, V. E. Morozov, S. V. Morozov, M. J. Zhu, S. L. Wong et al., Twist-controlled resonant tunneling in graphene/boron nitride/graphene heterostructures, Nat. Nanotechnol. 9, 808 (2014).
[59] U. Wurstbauer, B. Miller, E. Parzinger, and A. W. Holleitner, Light-matter interaction in transition metal dichalcogenides and their heterostructures, J. Phys. D: Appl. Phys. 50, 173001 (2017).

[60] T. Stroucken, J. H. Grönqvist, and S. W. Koch, Optical response and ground state of graphene, Phys. Rev. B 84, 205445 (2011).

[61] E. Malic, T. Winzer, E. Bobkin, and A. Knorr, Microscopic theory of absorption and ultrafast many-particle kinetics in graphene, Phys. Rev. B 84, 205406 (2011).

[62] J. W. McIver, B. Schulte, F.-U. Stein, T. Matsuyama, G. Jotzu, G. Meier, and A. Cavalleri, Light-induced anomalous Hall effect in graphene, Nat. Phys. 16, 38 (2020).

[63] A. Schiffrin, T. Paasch-Colberg, N. Karpowicz, V. Apalkov, D. Gerster, S. Mühlbrandt, M. Korbman, J. Reichert, M. Schultze, S. Holzner et al., Optical-field-induced current in dielectrics, Nature (London) 493, 70 (2013).

[64] S. A. Sato, J. W. McIver, M. Nuske, P. Tang, G. Jotzu, B. Schulte, H. Hübener, U. De Giovannini, L. Mathey, M. A. Sentef, A. Cavalleri, and A. Rubio, Microscopic theory for the light-induced anomalous Hall effect in graphene, Phys. Rev. B 99, 214302 (2019).

[65] V. Novicenko, E. Anisimovas, and G. Juzeliunas, Floquet analysis of a quantum system with modulated periodic driving, Phys. Rev. A 95, 023615 (2017).

[66] J. C. Cuevas and E. Scheer, Molecular Electronics: An Introduction to Theory and Experiment, 1st ed. (World Scientific, Singapore, 2010).

[67] J. Rammer, Quantum Field Theory of Nonequilibrium States, 1st ed. (Cambridge University Press, New York, 2007).

[68] J. P. Hobson and W. A. Nierenberg, The statistics of a twodimensional, hexagonal net, Phys. Rev. 89, 662 (1953).

[69] S. Yuan, H. De Raedt, and M. I. Katsnelson, Modeling electronic structure and transport properties of graphene with resonant scattering centers, Phys. Rev. B 82, 115448 (2010).

[70] Y. Zhou and M. W. Wu, Optical response of graphene under intense terahertz fields, Phys. Rev. B 83, 245436 (2011).

[71] S. Y. Zhou, G.-H. Gweon, A. V. Fedorov, P. N. First, W. A. de Heer, D.-H. Lee, F. Guinea, A. H. Castro Neto, and A. Lanzara, Substrate-induced bandgap opening in epitaxial graphene, Nat. Mater. 6, 770 (2007).

[72] A. H. Castro Neto, F. Guinea, N. M. R. Peres, K. S. Novoselov, and A. K. Geim, The electronic properties of graphene, Rev. Mod. Phys. 81, 109 (2009)

[73] T. Mueller and E. Malic, Exciton physics and device application of two-dimensional transition metal dichalcogenide semiconductors, NPJ 2D Mater. Appl. 2, 29 (2018).

[74] J. C. Cuevas, A. Martín-Rodero, and A. Levy Yeyati, Hamiltonian approach to the transport properties of superconducting quantum point contacts, Phys. Rev. B 54, 7366 (1996).

[75] J. C. Cuevas and M. Fogelström, Quasiclassical description of transport through superconducting contacts, Phys. Rev. B 64, 104502 (2001). 